\title{
ON SINGULAR INTEGRAL AND MARTINGALE TRANSFORMS
}

\author{
STEFAN GEISS, STEPHEN MONTGOMERY-SMITH, AND \\ EERO SAKSMAN
}

\begin{abstract}
Linear equivalences of norms of vector-valued singular integral operators and vector-valued martingale transforms are studied. In particular, it is shown that the UMD-constant of a Banach space $X$ equals the norm of the real (or the imaginary) part of the BeurlingAhlfors singular integral operator, acting on $L_{X}^{p}\left(\mathbf{R}^{2}\right)$ with $p \in(1, \infty)$. Moreover, replacing equality by a linear equivalence, this is found to be the typical property of even multipliers. A corresponding result for odd multipliers and the Hilbert transform is given. As a corollary we obtain that the norm of the real part of the Beurling-Ahlfors operator equals $p^{*}-1$ with $p^{*}:=\max \{p,(p /(p-1))\}$, where the novelty is the lower bound.
\end{abstract}

\section{INTRODUCTION}

A Banach space $X$ is said to be a UMD-space ${ }^{1}$ provided that for all (equivalently, for some) $p \in(1, \infty)$ there is a constant $c_{p}>0$ such that

$$
\sup _{\alpha_{k} \in\{ \pm 1\}}\left\|\sum_{k=1}^{n} \alpha_{k} D_{k}\right\|_{L_{X}^{p}} \leq c_{p}\left\|\sum_{k=1}^{n} D_{k}\right\|_{L_{X}^{p}}
$$

for all $n \geq 1$ and all $X$-valued martingale difference sequences $\left(D_{k}\right)_{k=1}^{n}$. As $\mathrm{UMD}$-constant one usually takes $\operatorname{UMD}_{p}(X):=\inf c_{p}$. We refer to [11] and the references therein for an overview about the UMD-property. It is known that in the above definition the arbitrary martingale differences can be replaced by Walsh-Paley martingale differences and one gets the same constant (see e.g. [10, p. 12] and [20]; the definition of Walsh-Paley martingales is recalled in Section 2 below). The UMD-property was first investigated by Burkholder [7], who gave a geometric characterization for Banach spaces with the UMD-property. Together with McConnell, Burkholder established in [8] that the Hilbert transform

$$
\mathcal{H} f(x):=\frac{1}{\pi} \int_{\mathbf{R}} \frac{f(y)}{x-y} d y
$$

is bounded on $L_{X}^{p}(R), p \in(1, \infty)$, provided that $X$ has the UMD-property. A converse result was proved soon after by Bourgain [5], who showed that

2000 Mathematics Subject Classification. 60G46, 42B15 (Primary), 42B20, 46B09, 46B20 (Secondary).

Key words and phrases. UMD property, singular integrals, martingale transforms.

The first and the last author are supported by the Project \#110599 of the Academy of Finland.

${ }^{1} \mathrm{UMD}$ stands for unconditional martingale differences. 
the boundedness of the Hilbert transform on $L_{X}^{p}$ for some $p \in(1, \infty)$ implies the UMD-property for $X$. It is also known from [6] that the UMD-property implies the boundedness of all invariant singular integrals or (more generally) standard multiplier operators under some regularity assumptions. The importance of the UMD-property, especially in connection with PDE:s, is further evidenced by recent new results on operator valued singular integrals (R-boundedness) [26] and other developments.

It is natural to ask for the quantitative equivalence of the UMD-property and the boundedness of the vector-valued Hilbert transform. The proofs in [8] and [5] yield that there is a constant $C>0$ such that

$$
\frac{1}{C}\left(\mathrm{UMD}_{2}(X)\right)^{1 / 2} \leq\|\mathcal{H}\|_{L_{X}^{2}(\mathbf{R}) \rightarrow L_{X}^{2}(\mathbf{R})} \leq C\left(\operatorname{UMD}_{2}(X)\right)^{2} .
$$

The curious feature above is the quadratic equivalence of the norms in contrast to the linear dependence one would expect. A better than a quadratic equivalence obtained from alternative proofs is not known to the authors.

The previous discussion raises the question whether there is a linear equivalence in (2). We do not know whether this is true or not. However, in this paper we establish that the answer is positive if $\mathcal{H}$ is replaced by the Beurling-Ahlfors transform $\mathcal{B A}$ :

Theorem 1.1. For $p \in(1, \infty)$ and a real Banach space $X$ one has that

$$
\mathrm{UMD}_{p}(X)=\|\operatorname{Re}(\mathcal{B A})\|_{L_{X}^{p}\left(\mathbf{R}^{2}\right) \rightarrow L_{X}^{p}\left(\mathbf{R}^{2}\right)}=\|\operatorname{Im}(\mathcal{B A})\|_{L_{X}^{p}\left(\mathbf{R}^{2}\right) \rightarrow L_{X}^{p}\left(\mathbf{R}^{2}\right)}
$$

with

$$
\mathcal{B A} f(z):=-\frac{1}{\pi} \int_{\mathbf{C}} \frac{f(w)}{(z-w)^{2}} d m_{2}(w),
$$

where $m_{2}$ is the two-dimensional Lebesgue measure on the complex plane $\mathbf{C}$, that is identified with $\mathbf{R}^{2}$.

Note that $\operatorname{Re}(\mathcal{B A})=-I d-2 R_{1}^{2}$ and $\operatorname{Im}(\mathcal{B A})=2 R_{1} R_{2}$ where $R_{1}$ and $R_{2}$ are the first and second Riesz transform, respectively. Equality (3) carries some new information for norm estimates even in the scalar case, see Corollary 3.2 below and the remark after it.

The operator $\mathcal{B A}$ is sometimes called the two-dimensional Hilbert transform. It plays a fundamental role in the theory of quasi-conformal maps and in the theory of elliptic equations in the plane. Quite recently, in connection with the well-known Iwaniec conjecture, there has been many works devoted to the probabilistic approach to estimate the (scalar) $L^{p}$-norm of the Beurling-Ahlfors operator, see e.g. [1, 25, 2].

If we replace the equality in (3) by a linear equivalence with multiplicative constants, then the obtained property is shared by an extensive class of operators corresponding to homogeneous multipliers. In this context the crucial difference between the Hilbert transform and the Beurling-Ahlfors operator is the parity of their integral kernels: our main Theorem 3.1 shows that one may replace the real and imaginary part of $\mathcal{B A}$ in (3) by any smooth, homogeneous of order zero, and even Fourier multiplier operator if we allow multiplicative constants. On the other side, Theorem 4.1 in turn relates odd multipliers to the Hilbert transform. 
The proof of Theorem 1.1 is based on a modification of an argument of Bourgain and the already standard representation of suitable combinations of Riesz singular integrals as certain transforms of Ito-integrals, see $[16,1]$. In order to define a (wider) class of such transforms, let $W=\left(\left(W_{t}^{1}, \ldots, W_{t}^{d}\right)\right)_{t>0}$ be a standard $d$-dimensional Brownian motion. For a Banach space $\bar{X}$, $p \in(1, \infty)$, and a real $d \times d$ matrix $A$ we let $\left\|I d_{X} \mid(A, W)\right\|_{p}:=\inf c$, where the infimum is taken over all $c>0$ such that

$$
\left\|\int_{0}^{T} U \cdot d\left(A W_{t}\right)\right\|_{L_{X}^{p}} \leq c\left\|\int_{0}^{T} U \cdot d\left(W_{t}\right)\right\|_{L_{X}^{p}}
$$

for all $T \geq 0$ and certain $d$-tuples $U$ of $X$-valued processes taking values in a finite-dimensional subspace of $X$ (see Section 2). As a byproduct of our proofs we obtain in Theorem 3.1 that, if the matrix $A$ is symmetric and not a constant multiple of the identity matrix, then there is a constant $C=C(A)>0$, independent of the Banach space $X$, such that

$$
\frac{1}{C} \operatorname{UMD}_{p}(X) \leq\left\|I d_{X} \mid(A, W)\right\|_{p} \leq C \operatorname{UMD}_{p}(X) .
$$

In the proof of Theorem 1.1 (in fact, Theorem 3.1) we do not employ harmonic extensions of functions to the upper half plane as it was done originally by Gundy and Varopoulos [16] in their stochastic proof of the $L^{p}$-boundedness of the scalar Riesz transforms. Instead, we follow Bañuelos and Mendez-Hernandez [2] and use the space-time Brownian motion, which corresponds to the heat extension of functions. Earlier the use of heat extensions in norms estimates for singular integrals was initiated by Nazarov, Petermichl and Volberg in [21] and [25]. The use of the space-time Brownian motion in combination with our modified version of Bourgain techniques is one reason that enables us to obtain the equalities (3). In particular, for all $d \geq 2$ it holds that

$$
\operatorname{UMD}_{p}(X)=\left\|I d+2 R_{1}^{2}\right\|_{L_{X}^{p}\left(\mathbf{R}^{d}\right) \rightarrow L_{X}^{p}\left(\mathbf{R}^{d}\right)}=\left\|I d_{X} \mid\left(A_{s}, W\right)\right\|_{p}
$$

with

$$
A_{s}:=\left[\begin{array}{cc}
1 & 0 \\
0 & -1
\end{array}\right]
$$

As one specializes to the case $X=\mathbf{R}$ in Theorem 1.1 one obtains

Corollary 1.2. For $p \in(1, \infty)$ one has that

$$
\|\operatorname{Re}(\mathcal{B A})\|_{L^{p}\left(\mathbf{R}^{2}\right) \rightarrow L^{p}\left(\mathbf{R}^{2}\right)}=p^{*}-1 \quad \text { where } p^{*}:=\max \left(p, \frac{p}{p-1}\right) .
$$

This follows because Burkholder (see [9], [11, Theorem 14], [10, p.12]) established that $\operatorname{UMD}_{p}(\mathbf{R})=p^{*}-1$. One should also observe here, that it is well-known that the $L^{p}$-norm of scalar multipliers that preserve real-valued functions does not depend on whether one look at real or complex $L^{p}$-spaces. The upper bound in Corollary 1.2 is due to Volberg and Nazarov [25, Theorem 3.1] who applied Bellman function and heat kernel techniques in the proof (a space-time Brownian motion approach to [25, Theorem 3.1] was subsequently given in [2]). The real novelty in our Corollary 1.2 is the lower bound. As far as we know, this is the first instance where the exact value of the norm of a 'second order' Riesz transform (a non-trivial expression 
involving products of Riesz transforms) has been determined. Moreover, Corollary 1.2 has obvious interest in connection with the famous Iwaniec conjecture, which claims that the norm of the full complex Beurling-Ahlfors transform equals $p^{*}-1$.

The paper is organized as follows: Section 2 recalls necessary notation and contains preparatory material. Our main result, Theorem 3.1, which contains Theorem 1.1, (5), and (6) as special cases, is formulated and proved in Section 3. Section 4 treats the case of odd kernels and anti-symmetric matrix transforms of stochastic integrals.

The results in Sections 3 and 4 are formulated for tensor product operators $T_{m} \otimes S$, where $S: X \rightarrow Y$ is an arbitrary operator between two Banach spaces $X$ and $Y$, instead for the setting of identities $I d_{X}$ we have used so far in the introduction. The motivation for this are certain connections to the geometry of Banach spaces that are explained in Section 5.

We would like to thank Tuomas Hytönen for the careful reading of the manuscript. In his recent paper [17] he obtained results about the linear equivalence of norms of vector valued spectral multipliers and the UMDconstant, which are in the spirit of the results in this paper (but, as pointed out in [17], the basic singular integral operators like $\mathcal{B A}$ or $\mathcal{H}$ do not fall into the setting and scope of [17]).

\section{Definitions AND PRELIMINARY RESUlTS}

We shall denote by $\left\{e_{1}, \ldots, e_{d}\right\}$ the unit vectors of $\mathbf{R}^{d}$, by $|x|$ the euclidean norm of $x \in \mathbf{R}^{d}$, and will use $B(x, \delta):=\left\{y \in \mathbf{R}^{d}:|x-y| \leq \delta\right\}$ for $\delta \geq 0$. Moreover, the set of real $m \times n$ matrices is denoted by $M(m, n)$.

Vector-valued operators. In this paper $L(X, Y)$ stands for the linear and bounded operators between two real Banach spaces $X$ and $Y$, where $L(X):=$ $L(X, X)$. Given a $\sigma$-finite measure space $(M, \mu)$ and $p \in[1, \infty)$, the space of Bochner integrable random variables $L_{X}^{p}(M)=L_{X}^{p}(M, \mu)$ consists of all strongly measurable functions $f: M \rightarrow X$ such that there is a separable subspace $X_{0} \subseteq X$ with $f(M) \subseteq X_{0}$ and $\|f\|_{L_{X}^{p}}:=\left(\int_{M}\|f\|^{p} d \mu\right)^{1 / p}<\infty$. Given operators $S \in L(X, Y)$ and $T \in L\left(L^{p}(M, \mu)\right)$ the tensor product $T \otimes S$ can be defined through its action on simple functions, i.e.

$$
(T \otimes S)\left(\sum_{k=1}^{n} x_{k} \chi_{E_{k}}\right):=\sum_{k=1}^{n} S x_{k} T\left(\chi_{E_{k}}\right) .
$$

In case that the quantity

$$
\begin{aligned}
\left\|T \otimes S: L_{X}^{p}(M, \mu) \rightarrow L_{Y}^{p}(M, \mu)\right\| \\
\quad:=\sup \left\{\|(T \otimes S)(F)\|_{L_{Y}^{p}}:\|F\|_{L_{X}^{p}} \leq 1, F \text { simple function }\right\}
\end{aligned}
$$

is finite, the operator $T \otimes S$ extends to a bounded linear operator from $L_{X}^{p}(M, \mu)$ into $L_{Y}^{p}(M, \mu)$. As particular operators $T$ we use multipliers. The usage of operators $S$ instead of identities Id :X $\rightarrow X$ of Banach spaces $X$ might be seen as somehow artificial at this point. As already mentioned, the motivation for this slightly more general setting can be found in Section 
5 , but the reader may, if she or he so wishes, replace the operator $S$ by an identity in what follows.

Multipliers. A bounded complex valued function $m \in C^{\infty}\left(\mathbf{R}^{d} \backslash\{0\}\right), d \geq 1$, is called (smooth) multiplier. A multiplier $m$ is homogeneous (of order zero) if $m(\lambda \xi)=m(\xi)$ for $\xi \in \mathbf{R}^{d} \backslash\{0\}$ and $\lambda>0$. In this paper the term multiplier always refers to smooth and homogeneous multipliers. The multiplier $m$ is called even provided that $m(\xi)=m(-\xi)$ for $\xi \neq 0$ and odd if $m(\xi)=-m(-\xi)$. The operator $T_{m}: L^{2}\left(\mathbf{R}^{d}\right) \rightarrow L^{2}\left(\mathbf{R}^{d}\right)$ associated to $m$ is given by

$$
T_{m} f:=\mathcal{F}^{-1}(m \mathcal{F} f),
$$

where $\mathcal{F}$ stands for the Fourier transform

$$
(\mathcal{F} f)(\xi):=\int_{\mathbf{R}^{d}} \exp (-i\langle\xi, x\rangle) f(x) d x .
$$

It is easy to check that an even and real multiplier maps real-valued functions to real-valued functions, and the same is true for odd and purely imaginary multipliers. Consequently, to formulate our results for real Banach spaces, from now on we use the standing

Assumption 2.1. All multipliers are even and real, or odd and purely imaginary.

Let $A \in M(d, d)$ be invertible. By applying the simple identity

$$
T_{m \circ A} f(x)=\left(T_{m}\left(f \circ A^{T}\right)\right)\left(\left(A^{T}\right)^{-1} x\right)
$$

we deduce that composing a multiplier with a linear invertible map one does not change its norm, i.e.

$$
\left\|\mathrm{T}_{m \circ A} \otimes S: L_{X}^{p}\left(\mathbf{R}^{d}\right) \rightarrow L_{Y}^{p}\left(\mathbf{R}^{d}\right)\right\|=\left\|\mathrm{T}_{m} \otimes S: L_{X}^{p}\left(\mathbf{R}^{d}\right) \rightarrow L_{Y}^{p}\left(\mathbf{R}^{d}\right)\right\| .
$$

For a multiplier $m$ on $\mathbf{R}^{d}$ as above there is a corresponding discrete multiplier $\widetilde{m}$ that acts on functions defined on the $d$-dimensional torus $\mathbf{T}^{d}:=(-\pi, \pi]^{d}$ : for a finite trigonometric polynomial $f$ we let

$$
\left(T_{\widetilde{m}} f\right)(\theta):=\sum_{k \in \mathbf{Z}^{d}} \hat{f}(k) e^{i\langle k, \theta\rangle} m(k),
$$

where $\hat{f}(k):=(1 / 2 \pi)^{d} \int_{\mathbf{T}^{d}} e^{-i\langle k, \theta\rangle} f(\theta) d \theta$ and $m(0):=\omega_{d-1}^{-1} \int_{S^{d-1}} m(x) d x$ is the average over the boundary of the euclidean unit ball (remember that $m$ is homogeneous of order zero). It follows by Assumption 2.1 that in the above definition $T_{\widetilde{m}} f$ is real whenever $f$ is real. In the sequel it will be important that the norms of the corresponding multipliers are equal which will be stated in Lemma 2.2 below.

Lemma 2.2. Let $m$ be a smooth and homogeneous multiplier on $\mathbf{R}^{d}, d \geq 1$. Then, for any $p \in(1, \infty)$ and $S \in L(X, Y)$, it holds that

$$
\begin{aligned}
\left\|T_{m} \otimes S: L_{X}^{p}\left(\mathbf{R}^{d}\right) \rightarrow L_{Y}^{p}\left(\mathbf{R}^{d}\right)\right\| & =\left\|T_{\widetilde{m}} \otimes S: L_{X}^{p}\left(\mathbf{T}^{d}\right) \rightarrow L_{Y}^{p}\left(\mathbf{T}^{d}\right)\right\| \\
& =\left\|T_{\widetilde{m}} \otimes S: L_{X, 0}^{p}\left(\mathbf{T}^{d}\right) \rightarrow L_{Y}^{p}\left(\mathbf{T}^{d}\right)\right\|,
\end{aligned}
$$

where $L_{X, 0}^{p}\left(\mathbf{T}^{d}\right)$ stands for the functions in $L_{X}^{p}\left(\mathbf{T}^{d}\right)$ of mean zero. 
Proof. The first equality in the scalar case is essentially due to K. de Leeuw [19], see also [12]. The proof in the monograph [15, pp. 221-223] can be easily verified to carry over to the case considered here to yield the estimate

$$
\left\|T_{\widetilde{m}} \otimes S: L_{X}^{p}\left(\mathbf{T}^{d}\right) \rightarrow L_{Y}^{p}\left(\mathbf{T}^{d}\right)\right\| \leq\left\|T_{m} \otimes S: L_{X}^{p}\left(\mathbf{R}^{d}\right) \rightarrow L_{Y}^{p}\left(\mathbf{R}^{d}\right)\right\| .
$$

The lemma follows as soon as one has

$$
\left\|T_{m} \otimes S: L_{X}^{p}\left(\mathbf{R}^{d}\right) \rightarrow L_{Y}^{p}\left(\mathbf{R}^{d}\right)\right\| \leq\left\|T_{\widetilde{m}} \otimes S: L_{X, 0}^{p}\left(\mathbf{T}^{d}\right) \rightarrow L_{Y}^{p}\left(\mathbf{T}^{d}\right)\right\| .
$$

Observe that when computing the norm of $T_{m} \otimes S$ we may restrict ourselves to functions of type $f=\sum_{k=1}^{n} f_{k} x_{k}$ where $x_{k} \in X$ and the $f_{k} \in C_{0}^{\infty}\left(\mathbf{R}^{d}\right)$ have integral zero because these functions $f$ are dense in $L_{X}^{p}\left(\mathbf{R}^{d}\right)$. Then we can also follow the second part of the proof of the transference principle in [15] on pp. 223-225. One also verifies, that in the above proofs one may restrict to real valued scalar functions $f_{k}$, and as our multipliers preserve real-valued functions, the result remains true also for real Banach spaces $X, Y$.

Hilbert transform. The Hilbert transform was defined in (1). It corresponds to the multiplier $m(\xi)=-i \operatorname{sgn}(\xi)$, and maps, by definition, real valued functions to real valued functions. The corresponding discrete multiplier operator $T_{\widetilde{m}}$ is the well known conjugation operator $\widetilde{\mathcal{H}}: L_{p}(\mathbf{T}) \rightarrow L_{p}(\mathbf{T})$, $p \in(1, \infty)$, which can be also defined through its action on the trigonometric polynomials

$$
(\widetilde{\mathcal{H}} \sin (k \cdot))(\theta):=-\cos (k \theta) \quad \text { and } \quad(\mathcal{H} \cos (k \cdot))(\theta):=\sin (k \theta)
$$

for $k=1,2, \ldots$ and $\widetilde{\mathcal{H}} 1: \equiv 0$.

Beurling-Ahlfors transform and Riesz transforms. The $k$-th Riesztransform $R_{k}, k=1, \ldots, d$ is the multiplier operator on $\mathbf{R}^{d}$ corresponding to the multiplier $\xi_{k} /(i|\xi|)$. The Beurling-Ahlfors operator, defined through (4) in the introduction, corresponds to the multiplier $m_{B A}(\xi):=\frac{\xi_{1}-i \xi_{2}}{\xi_{1}+i \xi_{2}}$. It follows that

$$
\mathcal{B A}=R_{2}^{2}-R_{1}^{2}+2 i R_{1} R_{2}=: \operatorname{Re}(\mathcal{B A})+i \operatorname{Im}(\mathcal{B A}) .
$$

For our real valued setting we consider $\operatorname{Re}(\mathcal{B A})$ and $\operatorname{Im}(\mathcal{B A})$ separately and check by (7) that a rotation of the coordinates by the angle $\pi / 4$ transforms $\operatorname{Re}(\mathcal{B A})$ into $\operatorname{Im}(\mathcal{B A})$ and their norms coincide.

Martingale transforms. Given independent Bernoulli random variables $\varepsilon_{1}, \varepsilon_{2}, \ldots$, i.e. $\mathbf{P}\left(\varepsilon_{k}= \pm 1\right)=1 / 2$, and maps $d_{k}: \mathbf{R}^{k-1} \rightarrow X$, where $X$ is a Banach space and $d_{1}$ is constant, a sequence $\left(\varepsilon_{k} d_{k}\left(\varepsilon_{1}, \ldots, \varepsilon_{k-1}\right)\right)_{k \in I}$ with $I=\{1, \ldots, n\}$ or $I=\{1,2, \ldots\}$ is called Walsh-Paley martingale difference sequence. Given $S \in L(X, Y)$ and $p \in(1, \infty)$, we define $\operatorname{UMD}_{p}(S):=\inf c$, where the infimum is taken over all $c>0$ such that

$$
\left\|\sum_{k=1}^{n} \alpha_{k} S \varepsilon_{k} d_{k}\right\|_{L_{Y}^{p}} \leq c\left\|\sum_{k=1}^{n} \varepsilon_{k} d_{k}\right\|_{L_{X}^{p}}
$$


for all $X$-valued Walsh-Paley martingale difference sequences $\left(\varepsilon_{k} d_{k}\right)_{k=1}^{n}$, all $\alpha_{1}, \ldots, \alpha_{n} \in \mathbf{R}$ with $\left|\alpha_{k}\right| \leq 1$, and $n \geq 1$. It is well-known that by an easy extreme point argument the condition $\left|\alpha_{k}\right| \leq 1$ can be replaced by $\alpha_{k} \in\{-1,1\}$ and one gets the same constant $\operatorname{UMD}_{p}(S)$. The reader is also referred to [11] and the references therein for a more general overview about UMD-spaces.

Transforms for stochastic integrals. We recall the definition given in the introduction. Let $W=\left(\left(W_{t}^{1}, \ldots, W_{t}^{d}\right)\right)_{t \geq 0}$ be a $d$-dimensional standard Brownian motion with continuous paths for all $\omega \in \Omega$ and $W_{0} \equiv 0$, defined on a probability space $(\Omega, \mathcal{F}, \mathbb{P})$, where $\mathcal{F}$ is the completion of $\sigma\left(W_{t}: t \geq 0\right)$ and $\left(\mathcal{F}_{t}\right)_{t \geq 0}$ the augmentation of the natural filtration of $W$. Let $S \in L(X, Y)$, $p \in(1, \infty)$, and $A=\left[a_{k l}\right] \in M(d, d)$. Then $\|S \mid(A, W)\|_{p}:=\inf c$, such that

$$
\left\|\sum_{k=1}^{d} \sum_{l=1}^{d} a_{k l} \int_{0}^{T} S U_{t}^{k} d W_{t}^{l}\right\|_{L_{Y}^{p}} \leq c\left\|\sum_{k=1}^{d} \int_{0}^{T} U_{t}^{k} d W_{t}^{k}\right\|_{L_{X}^{p}}
$$

for all $T \geq 0$ and $\left(\mathcal{F}_{t}\right)_{t>0}$-adapted left-continuous processes of Radon random variables $U_{t}^{k}: \Omega \rightarrow X$ which have right-hand side limits, take values in a finite-dimensional subspace of $X$, satisfy

$$
\int_{0}^{T} \mathbb{E}\left\|U_{t}^{k}\right\|_{X}^{2} d t<\infty
$$

for all $T \geq 0$ and $k=1, \ldots, d$, and such that the right hand side of (9) is finite. To shorten the notation we also use in the sequel $\int_{0}^{T}\left(S U_{t}^{k}\right)_{k=1}^{d} \cdot d(A W)_{t}$ and $\int_{0}^{T}\left(U_{t}^{k}\right)_{k=1}^{d} \cdot d W_{t}$, respectively, for the expressions inside the norms.

Some minor notation. Given $A, B \geq 0$ and $c>0$, the notation $A \sim_{c} B$ stands for $A / c \leq B \leq c A$. If the dependence of $c$ on the extra quantities involved is clear, we sometimes simply write $A \sim B$.

\section{The MAin Result}

It will be convenient to have a special notation for particular matrices and multipliers. Thus, we denote

$$
\mathrm{A}_{\mathrm{s}}:=\left[\begin{array}{cc}
1 & 0 \\
0 & -1
\end{array}\right], \quad A_{\mathrm{s}, d}:=\left[\begin{array}{ccccc}
1 & 0 & 0 & \ldots & 0 \\
0 & -1 & 0 & \ldots & 0 \\
0 & 0 & -1 & \ldots & 0 \\
\vdots & \vdots & \vdots & \ddots & \vdots \\
0 & 0 & 0 & 0 & -1
\end{array}\right], \quad \mathrm{A}_{\mathrm{as}}:=\left[\begin{array}{cc}
0 & -1 \\
1 & 0
\end{array}\right] .
$$

We shall also define the special multiplier $m_{0}$ on $\mathbf{R}^{d}$, where $d \geq 2$, by

$$
m_{0}(\xi):=2 \frac{\xi_{1}^{2}}{|\xi|^{2}}-1, \quad \text { equivalently } \quad T_{m_{0}}:=-\left(\operatorname{Id}+2 R_{1}^{2}\right) .
$$

We recall once more that, for simplicity, all Banach spaces are assumed to be real. Our main result is the following: 
Theorem 3.1. Assume that $m \in C^{\infty}\left(\mathbf{R}^{d} \backslash\{0\}\right), d \geq 2$, is a real, homogeneous, and even multiplier that is not identically constant, and $A \in M(d, d)$ is a real symmetric matrix that is not a multiple of the identity matrix. Let $p \in(1, \infty)$. Then there is a constant $C=C(m, A)$ such that for every pair of Banach spaces $X$ and $Y$ and for every operator $S \in L(X, Y)$ one has that

$$
\begin{aligned}
\left\|S \mid\left(\mathrm{A}_{\mathrm{s}}, W\right)\right\|_{p}=\operatorname{UMD}_{p}(S)=\left\|T_{m_{0}} \otimes S: L_{X}^{p}\left(\mathbf{R}^{d}\right) \rightarrow L_{Y}^{p}\left(\mathbf{R}^{d}\right)\right\| \\
\quad \sim_{C}\left\|T_{m} \otimes S: L_{X}^{p}\left(\mathbf{R}^{d}\right) \rightarrow L_{Y}^{p}\left(\mathbf{R}^{d}\right)\right\| \sim_{C}\|S \mid(A, W)\|_{p} .
\end{aligned}
$$

Exploiting $\operatorname{UMD}_{p}(\mathbf{R})=p-1$ for $p \in[2, \infty)$ (see [9], [11, Theorem 14]) and using that, for $d=2$, the multiplier $m_{0}$ corresponds to $R_{2}^{2}-R_{1}^{2}$ and $2 R_{1} R_{2}$ can be obtained by a rotation of $m_{0}$, Theorem 3.1 implies

Corollary 3.2. For $d=2, p \in(1, \infty)$, and $S \in L(X, Y)$ one has

$$
\begin{aligned}
\left\|\operatorname{Re}(\mathcal{B A}) \otimes S: L_{X}^{p}\left(\mathbf{R}^{2}\right) \rightarrow L_{Y}^{p}\left(\mathbf{R}^{2}\right)\right\| \\
\quad=\left\|\operatorname{Im}(\mathcal{B A}) \otimes S: L_{X}^{p}\left(\mathbf{R}^{2}\right) \rightarrow L_{Y}^{p}\left(\mathbf{R}^{2}\right)\right\|=\operatorname{UMD}_{p}(S) .
\end{aligned}
$$

In particular, for $p \in[2, \infty)$,

$$
\left\|\operatorname{Re}(\mathcal{B A}): L^{p}\left(\mathbf{R}^{2}\right) \rightarrow L^{p}\left(\mathbf{R}^{2}\right)\right\|=\left\|\operatorname{Im}(\mathcal{B A}): L^{p}\left(\mathbf{R}^{2}\right) \rightarrow L^{p}\left(\mathbf{R}^{2}\right)\right\|=p-1 .
$$

We break the proof of Theorem 3.1 into a series of auxiliary results, some of which are basically known, and some of which may have independent interest. The actual proof of Theorem 3.1 is given at the end of this section. Our first step is to show that non-trivial even multipliers dominate the UMD-constant linearly. To this end we need a generalization of a Lemma due to Bourgain [5, Lemma 1]. For the rest of this section we assume that $X$ and $Y$ are Banach spaces and $S \in L(X, Y)$ is a fixed operator.

Lemma 3.3. Let $p \in(1, \infty), Q:=\mathbf{T}^{d}$, and assume that the multiplier $m$ satisfies Assumption 2.1. For $k \geq 1$ let $E_{k}$ be the closure in $L_{X}^{p}\left(Q^{k}\right)$ of the finite real trigonometric polynomials

$$
\Phi_{k}\left(\theta_{1}, \ldots, \theta_{k}\right)=\sum_{p=1}^{P} \Phi_{k}^{p}\left(\theta_{1}, \ldots, \theta_{k}\right) x_{p}
$$

with $x_{k} \in X$ and

$$
\Phi_{k}^{p}=\sum_{\ell_{1} \in \mathbf{Z}^{d}} \cdots \sum_{\ell_{k} \in \mathbf{Z}^{d}} e^{i\left\langle\ell_{1}, \theta_{1}\right\rangle} \cdots e^{i\left\langle\ell_{k}, \theta_{k}\right\rangle} \alpha_{\ell_{1}, \ldots, \ell_{k}}^{p} \quad \text { where } \quad \operatorname{Im}\left(\Phi_{k}^{p}\right) \equiv 0
$$

where only finitely many of the $\alpha_{\ell_{1}, \ldots, \ell_{k}}^{p} \in \mathbf{C}$ are non-zero and $\alpha_{\ell_{1}, \ldots, \ell_{k}}^{p}=0$ whenever $\ell_{k}=0$ (so that $\int_{Q} \Phi_{k}^{p}\left(\theta_{1}, \ldots, \theta_{k}\right) d \theta_{k}=0$ ). Let $T_{\tilde{m}}^{k}: L^{p}\left(Q^{k}\right) \rightarrow$ $L^{p}\left(Q^{k}\right)$ be given by

$$
\begin{aligned}
& \left(T_{\widetilde{m}}^{k} \Phi_{k}\right)\left(\theta_{1}, \ldots, \theta_{k}\right) \\
& \quad=\sum_{p=1}^{P}\left(\sum_{\ell_{1} \in \mathbf{Z}^{d}} \cdots \sum_{\ell_{k} \in \mathbf{Z}^{d}} m\left(\ell_{k}\right) e^{i\left\langle\ell_{1}, \theta_{1}\right\rangle} \ldots e^{i\left\langle\ell_{k}, \theta_{k}\right\rangle} \alpha_{\ell_{1}, \ldots, \ell_{k}}^{p}\right) x_{p} .
\end{aligned}
$$


for $\ell_{1}, \ldots, \ell_{k} \in \mathbf{Z}^{d}$. Then one has that

$$
\begin{aligned}
& \left\|\sum_{k=1}^{n}\left(\left(T_{\widetilde{m}}^{k} \otimes S\right) \Phi_{k}\right)\left(\theta_{1}, \ldots, \theta_{k}\right)\right\|_{L_{Y}^{p}\left(Q^{n}\right)} \\
& \quad \leq\left\|T_{\widetilde{m}} \otimes S: L_{X}^{p}\left(\mathbf{T}^{d}\right) \rightarrow L_{Y}^{p}\left(\mathbf{T}^{d}\right)\right\|\left\|\sum_{k=1}^{n} \Phi_{k}\left(\theta_{1}, \ldots, \theta_{k}\right)\right\|_{L_{X}^{p}\left(Q^{n}\right)}
\end{aligned}
$$

for $\Phi_{1} \in E_{1}, \ldots, \Phi_{n} \in E_{n}$.

Proof. It is sufficient to prove the inequality for finite real trigonometric polynomials $\Phi_{1}\left(\theta_{1}\right), \ldots, \Phi_{n}\left(\theta_{1}, \ldots, \theta_{n}\right)$. Let $A \geq 1$ be an integer, $\eta \in Q$ be an auxiliary variable, denote by $T_{\widetilde{m}, \eta}$ the application of $T_{\widetilde{m}}$ with respect to the variable $\eta$, and consider the difference

$$
\begin{aligned}
D_{k}^{A}\left(\theta_{1}, \ldots, \theta_{k}, \eta\right):= & \left(\left(T_{\widetilde{m}, \eta} \otimes S\right) \Phi_{k}\left(\theta_{1}+A \cdot, \ldots, \theta_{k}+A^{k} \cdot\right)\right)(\eta) \\
& -\left(\left(T_{\widetilde{m}}^{k} \otimes S\right) \Phi_{k}\right)\left(\theta_{1}+A \eta, \ldots, \theta_{k}+A^{k} \eta\right) .
\end{aligned}
$$

Note, that in the first term on the right-hand side we apply the multiplier $T_{\widetilde{m}}$ to a function, where $\theta_{1}, \ldots, \theta_{k}, A$ act as parameters, whereas in the second term we apply the multiplier $T_{\tilde{m}}^{k}$ to $\Phi_{k}$ itself. If we show that for our fixed trigonometric polynomial $\Phi_{k}$ there is the bound

$$
\sup _{A \geq 1} A\left\|D_{k}^{A}\right\|_{L_{Y}^{\infty}}<\infty
$$

then the proof is completed by, firstly, observing that

$$
\begin{aligned}
& \left\|\sum_{k=1}^{n}\left(\left(T_{\widetilde{m}, \eta} \otimes S\right) \Phi_{k}\left(\theta_{1}+A \cdot, \ldots, \theta_{k}+A^{k} \cdot\right)\right)(\eta)\right\|_{L_{Y}^{p}(Q, d \eta)} \\
\leq & \left\|T_{\widetilde{m}} \otimes S: L_{X}^{p}(Q) \rightarrow L_{Y}^{p}(Q)\right\|\left\|\sum_{k=1}^{n} \Phi_{k}\left(\theta_{1}+A \eta, \ldots, \theta_{k}+A^{k} \eta\right)\right\|_{L_{X}^{p}(Q, d \eta)} .
\end{aligned}
$$

Secondly, one replaces the left-hand side by

$$
\left\|\sum_{k=1}^{n}\left(\left(T_{\widetilde{m}}^{k} \otimes S\right) \Phi_{k}\right)\left(\theta_{1}+A \eta, \ldots, \theta_{k}+A^{k} \eta\right)\right\|_{L_{Y}^{p}(Q, d \eta)}
$$

with the corresponding correction terms in $A$, integrates with respect to the $\theta$ 's, applies Fubini's theorem so that the variable $\eta$ is removed, and sends $A$ to infinity.

In order to prove (11) we observe that $A\left\|D_{k}^{A}\left(\theta_{1}, \ldots, \theta_{k}, \eta\right)\right\|_{Y}$ is upper bounded by a finite number of terms of form

$$
\begin{aligned}
A \mid T_{\widetilde{m}, \eta}\left(e^{i\left\langle\ell_{1}, \theta_{1}+A \eta\right\rangle}\right. & \left.\cdots e^{i\left\langle\ell_{k}, \theta_{k}+A^{k} \eta\right\rangle}\right) \\
& -m\left(\ell_{k}\right) e^{i\left\langle\ell_{1}, \theta_{1}+A \eta\right\rangle} \cdots e^{i\left\langle\ell_{k}, \theta_{k}+A^{k} \eta\right\rangle}|| \alpha_{\ell_{1}, \ldots, \ell_{k}}^{p} \mid\left\|S x_{p}\right\|_{Y}
\end{aligned}
$$

with $\ell_{k} \neq 0$. Finally we observe that

$$
A \mid T_{\widetilde{m}, \eta}\left(e^{i\left\langle\ell_{1}, \theta_{1}+A \eta\right\rangle} \cdots e^{i\left\langle\ell_{k}, \theta_{k}+A^{k} \eta\right\rangle}\right)
$$




$$
\begin{aligned}
& -m\left(\ell_{k}\right) e^{i\left\langle\ell_{1}, \theta_{1}+A \eta\right\rangle} \cdots e^{i\left\langle\ell_{k}, \theta_{k}+A^{k} \eta\right\rangle} \mid \\
= & A\left|m\left(\ell_{1} A+\ldots+\ell_{k} A^{k}\right)-m\left(\ell_{k}\right)\right| \\
= & A\left|m\left(\ell_{1} A^{-k+1}+\ldots+\ell_{k-1} A^{-1}+\ell_{k}\right)-m\left(\ell_{k}\right)\right|
\end{aligned}
$$

which is bounded in $A$ since $m$ is differentiable at $\ell_{k} \neq 0$. This yields (11). $\square$

Proposition 3.4. Assume that $m \in C^{\infty}\left(\mathbf{R}^{d} \backslash\{0\}\right), d \geq 2$, is a smooth, homogeneous, even, and non-constant multiplier. Let $\delta^{+}:=\max _{|\xi|=1} m(\xi)$ and $\delta^{-}:=\min _{|\xi|=1} m(\xi)$ so that $\delta^{+}-\delta^{-}>0$. Then

$$
\operatorname{UMD}_{p}(S) \leq \frac{2}{\delta^{+}-\delta^{-}}\left(1+\frac{\left|\delta^{+}+\delta^{-}\right|}{\left|\delta^{+}\right|+\left|\delta^{-}\right|}\right)\left\|T_{m} \otimes S: L_{X}^{p}\left(\mathbf{R}^{d}\right) \rightarrow L_{Y}^{p}\left(\mathbf{R}^{d}\right)\right\| .
$$

In particular, in the case $\max _{|\xi|=1} m(\xi)=-\min _{|\xi|=1} m(\xi)=1$ we have that

$$
\operatorname{UMD}_{p}(S) \leq\left\|T_{m} \otimes S: L_{X}^{p}\left(\mathbf{R}^{d}\right) \rightarrow L_{Y}^{p}\left(\mathbf{R}^{d}\right)\right\| .
$$

Proof. By continuity and compactness there are $\xi^{-}, \xi^{+} \in \mathbf{R}^{d}$ of length one such that $m\left(\xi^{-}\right)=\delta^{-}$and $m\left(\xi^{+}\right)=\delta^{+}$. Without loss of generality we may assume that $\xi^{-}=e_{1}$ and $\xi^{+}=e_{2}$, where $e_{1}$ and $e_{2}$ are the first two unit vectors in $\mathbf{R}^{d}$ (otherwise (7) enables us to replace $m(\xi)$ by $m(A \xi)$ with suitably chosen $A$ ). Define the functions $a^{-}, a^{+} \in L^{\infty}\left(\mathbf{T}^{d}\right)$ by $a^{-}(\theta):=$ $\operatorname{sgn}\left(\theta_{1}\right)$ and $a^{+}(\theta):=\operatorname{sgn}\left(\theta_{2}\right)$ for $\theta \in \mathbf{T}^{d}$ so that $T_{\widetilde{m}} a^{-}=\delta^{-} a^{-}$and $T_{\widetilde{m}} a^{+}=$ $\delta^{+} a^{+}$. For independent Bernoulli random variables $\varepsilon_{1}, \varepsilon_{2}, \ldots$ we consider the $X$-valued Walsh-Paley martingale difference sequence

$$
\left(\varepsilon_{k} d_{k}\left(\varepsilon_{1}, \ldots, \varepsilon_{k-1}\right)\right)_{k=1}^{n}
$$

and a sequence $\left(\alpha_{k}\right)_{k=1}^{n}$ with $\alpha_{k} \in\left\{\delta^{-}, \delta^{+}\right\}$. Define $\psi_{k}:=a^{-}$if $\alpha_{k}=\delta^{-}$ and $\psi_{k}:=a^{+}$if $\alpha_{k}=\delta^{+}$, and let

$$
\phi_{k}\left(\theta_{1}, \ldots, \theta_{k-1}\right):=d_{k}\left(\psi_{1}\left(\theta_{1}\right), \ldots, \psi_{k-1}\left(\theta_{k-1}\right)\right) .
$$

Since $\left(\psi_{1}\left(\theta_{1}\right), \ldots, \psi_{n}\left(\theta_{n}\right)\right)$ and $\left(\varepsilon_{1}, \ldots, \varepsilon_{n}\right)$ have the same distribution (if we normalize the measure on $\left.Q^{n}\right)$ and $T_{\widetilde{m}} \psi_{k}=\alpha_{k} \psi_{k}$, Lemma 3.3 implies that

$$
\begin{aligned}
& \left\|\sum_{k=1}^{n} \alpha_{k} \varepsilon_{k} S_{k} d_{k}\left(\varepsilon_{1}, \ldots, \varepsilon_{k-1}\right)\right\| \\
& \quad \leq\left\|T_{\widetilde{m}} \otimes S: L_{X}^{p}\left(\mathbf{T}^{d}\right) \rightarrow L_{Y}^{p}\left(\mathbf{T}^{d}\right)\right\| \| \sum_{k=1}^{n} \varepsilon_{k} d_{k}\left(\varepsilon_{1}, \ldots, \varepsilon_{k-1}\right) \\
& \quad \|_{L_{X}^{p}} .
\end{aligned}
$$

Let $A:=2 /\left(\delta^{+}-\delta^{-}\right)$and $B:=\left(\delta^{+}+\delta^{-}\right) /\left(\delta^{+}-\delta^{-}\right)$so that the new sequence $\beta_{k}:=A \alpha_{k}-B$ satisfies $\beta_{k}=-1$ if $\alpha_{k}=\delta^{-}$and $\beta_{k}=1$ if $\alpha_{k}=\delta^{+}$. Then

$$
\left\|\sum_{k=1}^{n} \beta_{k} \varepsilon_{k} S_{k} d_{k}\left(\varepsilon_{1}, \ldots, \varepsilon_{k-1}\right)\right\|_{L_{Y}^{p}}
$$




$$
\leq\left[A\left\|T_{\widetilde{m}} \otimes S: L_{X}^{p}\left(\mathbf{T}^{d}\right) \rightarrow L_{Y}^{p}\left(\mathbf{T}^{d}\right)\right\|+|B|\|S\|\right]\left\|\sum_{k=1}^{n} \varepsilon_{k} d_{k}\left(\varepsilon_{1}, \ldots, \varepsilon_{k-1}\right)\right\|_{L_{X}^{p}} .
$$

Because $\|m\|_{\infty}\|S\|=\sup _{\ell \in \mathbf{Z}^{d}}|m(\ell)|\|S\| \leq\left\|T_{\widetilde{m}} \otimes S: L_{X}^{p}\left(\mathbf{T}^{d}\right) \rightarrow L_{Y}^{p}\left(\mathbf{T}^{d}\right)\right\|$ we end up with

$$
\begin{aligned}
\operatorname{UMD}_{p}(S) & \leq\left[A+\frac{|B|}{\|m\|_{\infty}}\right]\left\|T_{\widetilde{m}} \otimes S: L_{X}^{p}\left(\mathbf{T}^{d}\right) \rightarrow L_{Y}^{p}\left(\mathbf{T}^{d}\right)\right\| \\
& =\left[A+\frac{|B|}{\|m\|_{\infty}}\right]\left\|T_{m} \otimes S: L_{X}^{p}\left(\mathbf{R}^{d}\right) \rightarrow L_{Y}^{p}\left(\mathbf{R}^{d}\right)\right\| \\
& \leq \frac{2}{\delta^{+}-\delta^{-}}\left(1+\frac{\left|\delta^{+}+\delta^{-}\right|}{\left|\delta^{+}\right|+\left|\delta^{-}\right|}\right)\left\|T_{m} \otimes S: L_{X}^{p}\left(\mathbf{R}^{d}\right) \rightarrow L_{Y}^{p}\left(\mathbf{R}^{d}\right)\right\|
\end{aligned}
$$

where the equality follows from Lemma 2.2 .

Proposition 3.5. For $T_{m_{0}}=-\mathrm{Id}-2 R_{1}^{2}, p \in(1, \infty)$, and $d \geq 2$ one has

$$
\left\|T_{m_{0}} \otimes S: L_{X}^{p}\left(\mathbf{R}^{d}\right) \rightarrow L_{Y}^{p}\left(\mathbf{R}^{d}\right)\right\| \leq\left\|S \mid\left(A_{\mathrm{s}, d}, W\right)\right\|_{p} .
$$

Proof. We apply the representation of products of Riesz-transforms in terms of heat extensions to the upper half space (see Lemma 6.1). Let $f=\sum_{k=1}^{m} f_{k} x_{k}$ and $g=\sum_{l=1}^{n} g_{l} b_{l}$ with $f_{k}, g_{l} \in C_{0}^{\infty}\left(\mathbf{R}^{d}\right)$ and $x_{k} \in X$, $b_{l} \in Y^{\prime}$. Assume that $u_{k}$ and $v_{l}$ are the heat extensions of $f_{k}$ and $g_{l}$, respectively, to the upper half plane and that $\left(W_{t}\right)_{t \geq 0}$ is a standard Brownian motion in $\mathbf{R}^{d}$ starting in the origin. Let $u:=\sum_{k=1}^{m} u_{k} x_{k}, v:=\sum_{l=1}^{n} v_{l} b_{l}$, and $1=(1 / p)+\left(1 / p^{\prime}\right)$. Lemma 6.1 gives that

$$
\begin{aligned}
& \left|\int_{\mathbf{R}^{d}}\left\langle\left(\left(T_{m_{0}} \otimes S\right) f\right)(x), g(x)\right\rangle d x\right| \\
& =\lim _{T \rightarrow \infty}(2 \pi T)^{d / 2} \mid \sum_{k, l}\left\langle S x_{k}, b_{l}\right\rangle \times \\
& \quad \times \mathbf{E}\left(\int_{0}^{T} \nabla u_{k}\left(W_{t}, T-t\right) \cdot d\left(A_{\mathrm{s}, d} W\right)_{t} \int_{0}^{T} \nabla v_{l}\left(W_{t}, T-t\right) \cdot d W_{t}\right) \mid \\
& =\lim _{T \rightarrow \infty}(2 \pi T)^{d / 2} \\
& \quad\left|\mathbf{E}\left\langle\int_{0}^{T} \nabla S u\left(W_{t}, T-t\right) \cdot d\left(A_{\mathrm{s}, d} W\right)_{t}, \int_{0}^{T} \nabla v\left(W_{t}, T-t\right) \cdot d W_{t}\right\rangle\right| .
\end{aligned}
$$

We continue with

$$
\begin{gathered}
\left|\mathbf{E}\left\langle\int_{0}^{T} \nabla S u\left(W_{t}, T-t\right) \cdot d\left(A_{\mathrm{s}, d} W\right)_{t}, \int_{0}^{T} \nabla v\left(W_{t}, T-t\right) \cdot d W_{t}\right\rangle\right| \\
\left.\leq \mid \mathbf{E}\left\|\int_{0}^{T} \nabla S u\left(W_{t}, T-t\right) \cdot d\left(A_{\mathrm{s}, d} W\right)_{t}\right\|_{Y}^{p}\right)^{1 / p} \\
\times\left(\mathbf{E}\left\|\int_{0}^{T} \nabla v\left(W_{t}, T-t\right) \cdot d W_{t}\right\|_{Y^{\prime}}^{p^{\prime}}\right)^{1 / p^{\prime}}
\end{gathered}
$$




$$
\begin{gathered}
\leq\left\|S \mid\left(A_{\mathrm{s}, d}, W\right)\right\|_{p}\left(\mathbf{E}\left\|\int_{0}^{T} \nabla u\left(W_{t}, T-t\right) \cdot d W_{t}\right\|_{Y}^{p}\right)^{1 / p} \\
\quad \times\left(\mathbf{E}\left\|\int_{0}^{T} \nabla v\left(W_{t}, T-t\right) \cdot d W_{t}\right\|_{Y^{\prime}}^{p^{\prime}}\right)^{1 / p^{\prime}} \\
=\left\|S \mid\left(A_{\mathrm{s}, d}, W\right)\right\|_{p}\left\|f\left(W_{T}\right)-u(0, T)\right\|_{L_{Y}^{p}}\left\|g\left(W_{T}\right)-v(0, T)\right\|_{L_{Y^{\prime}}^{p^{\prime}}}
\end{gathered}
$$

by Itô's formula because $(1 / 2) \Delta u_{k}=(\partial / \partial t) u_{k}$ and $(1 / 2) \Delta v_{l}=(\partial / \partial t) v_{l}$. Next, $\sup _{T>0}\left\|T^{d / 2} u(0, T)\right\|_{X}<\infty$ gives $\lim _{T \rightarrow \infty} T^{d / 2}\|u(0, T)\|_{X}^{p}=0$ so that $\lim _{T \rightarrow \infty}(2 \pi T)^{d / 2} \mathbf{E}\left\|f\left(W_{T}\right)-u(0, T)\right\|_{X}^{p}=\|f\|_{L_{X}^{p}\left(\mathbf{R}^{d}\right)}^{p}$. The same applies for $g\left(W_{T}\right)$ and we end up with

$$
\left|\int_{\mathbf{R}^{d}}\left\langle\left(\left(T_{m_{0}} \otimes S\right) f\right)(x), g(x)\right\rangle d x\right| \leq\left\|S \mid\left(A_{\mathrm{s}, d}, W\right)\right\|\left\|_{p}\right\| f\left\|_{L_{X}^{p}\left(\mathbf{R}^{d}\right)}\right\| g \|_{L_{Y^{\prime}}^{p^{\prime}}\left(\mathbf{R}^{d}\right)} .
$$

The proof is complete because $f$ and $g$ as above are dense in $L_{X}^{p}\left(\mathbf{R}^{d}\right)$ and $L_{Y^{\prime}}^{p^{\prime}}\left(\mathbf{R}^{d}\right)$, respectively.

In order to exploit the quantities $\|S \mid(A, W)\|_{p}$ in rigorous arguments, one needs at some places approximations of stochastic integrals by discrete martingales. So for the general reader's convenience we switch to the simple discretized version $\|S \mid(A, g)\|_{p}$ introduced below. Just to prove part of our main result one could proceed more directly (see Remark 3.10). Given $p \in$ $(1, \infty), S \in L(X, Y)$, and $A \in M(d, d)$, we let

$$
\|S \mid(A, g)\|_{p}:=\inf c,
$$

where the infimum is taken over all $c>0$ such that

$$
\begin{array}{r}
\left\|\sum_{k=1}^{N} \sum_{l=1}^{d}\left[S d_{(k-1) d+l}\left(\varphi_{1}, \ldots, \varphi_{(k-1) d}\right)\right]\left\langle A\left(\varphi_{(k-1) d+1}, \ldots, \varphi_{k d}\right), e_{l}\right\rangle\right\|_{L_{Y}^{p}} \\
\leq c\left\|\sum_{k=1}^{N} \sum_{l=1}^{d}\left[d_{(k-1) d+l}\left(\varphi_{1}, \ldots, \varphi_{(k-1) d}\right)\right] \varphi_{(k-1) d+l}\right\|_{L_{X}^{p}},
\end{array}
$$

where $N=1,2, \ldots, d_{j}: \mathbf{R}^{\left\lfloor\frac{j-1}{d}\right\rfloor d} \rightarrow X$ are continuous bounded functions taking values in a finite dimensional subspace of $X$, and $\varphi_{1}, \varphi_{2}, \ldots$ are independent standard Gaussian random variables. The $N d$ terms on the right-hand side (in their natural order) we call a Gaussian block martingale difference sequence of order $d \geq 1$, on the left-hand side we have its $A$-transform. Observe that these transforms are not the traditional martingale transforms appearing in the definition of the UMD-spaces.

Lemma 3.6. Let $A=\left[a_{l k}\right] \in M(d, d), S \in L(X, Y)$, and $p \in(1, \infty)$. Then the following is true:

(i) $\left\|S\left|(A, g)\left\|_{p}=\right\| S\right|(A, W)\right\|_{p}$.

(ii) If $U \in M(d, d)$ is real and unitary, then

$$
\left\|S\left|\left(U^{T} A U, g\right)\left\|_{p}=\right\| S\right|(A, g)\right\|_{p} .
$$


(iii) If $M \geq 1$ is an integer and the tensor product $\otimes^{M} A$ is defined as the block diagonal matrix with $A$ as each diagonal block, then

$$
\left\|S\left|\left(\otimes^{M} A, g\right)\left\|_{p}=\right\| S\right|(A, g)\right\|_{p} .
$$

(iv) Assume a sub-matrix $B$ of $A$ obtained from $A$ by choosing indices $I=\left\{k_{1}, \ldots, k_{d^{\prime}}\right\}$ with $1 \leq k_{1}<k_{2}<\ldots<k_{d^{\prime}} \leq d$ and $1 \leq d^{\prime}<d$, and deleting the corresponding rows and columns from $A$. Then

$$
\left\|S\left|(B, g)\left\|_{p} \leq\right\| S\right|(A, g)\right\|_{p} .
$$

Proof. (i) It is evident that $\left\|S\left|(A, g)\left\|_{p} \leq\right\| S\right|(A, W)\right\|_{p}$. The inequality to the other direction follows by a standard approximation of Ito-integrals by discrete Gaussian martingales.

(ii) Here we observe that

$$
\begin{aligned}
& \left\|\sum_{k=1}^{N} \sum_{l=1}^{d}\left[S d_{(k-1) d+l}\left(\varphi_{1}, \ldots, \varphi_{(k-1) d}\right)\right]\left\langle U^{T} A U\left(\varphi_{(k-1) d+1}, \ldots, \varphi_{k d}\right), e_{l}\right\rangle\right\|_{L_{Y}^{p}} \\
= & \left\|\sum_{k=1}^{N}\left\langle U\left(\left(S d_{(k-1) d+l}\left(\varphi_{1}, \ldots, \varphi_{(k-1) d}\right)\right)_{l=1}^{d}\right), A U\left(\varphi_{(k-1) d+1}, \ldots, \varphi_{k d}\right)\right\rangle\right\|_{L_{Y}^{p}} \\
\leq & \|S \mid(A, g)\|_{p} \cdot \\
& \left\|\sum_{k=1}^{N}\left\langle U\left(\left(d_{(k-1) d+l}\left(\varphi_{1}, \ldots, \varphi_{(k-1) d}\right)\right)_{l=1}^{d}\right), U\left(\varphi_{(k-1) d+1}, \ldots, \varphi_{k d}\right)\right\rangle\right\|_{L_{Y}^{p}} \\
= & \|S \mid(A, g)\|_{p}\left\|\sum_{k=1}^{N} \sum_{l=1}^{d}\left[d_{(k-1) d+l}\left(\varphi_{1}, \ldots, \varphi_{(k-1) d}\right)\right] \varphi_{(k-1) d+l}\right\|_{L_{X}^{p}} .
\end{aligned}
$$

Above we used in the second step the observation that the transformed sequence

$$
\begin{aligned}
& \left\langle U\left(\varphi_{1}, \ldots, \varphi_{d}\right), e_{1}\right\rangle, \ldots,\left\langle U\left(\varphi_{1}, \ldots, \varphi_{d}\right), e_{d}\right\rangle \\
& \left\langle U\left(\varphi_{d+1}, \ldots, \varphi_{2 d}\right), e_{1}\right\rangle, \ldots,\left\langle U\left(\varphi_{d+1}, \ldots, \varphi_{2 d}\right), e_{d}\right\rangle, \ldots
\end{aligned}
$$

consists again of independent Gaussian random variables because $U$ is unitary.

(iii) The $\otimes^{M} A$-transform of an appropriate Gaussian martingale block difference sequence of order $d M$ is obtained by simply performing the $A$ transform of the same sequence (which is also of order $d$ ). This shows that $\left\|S\left|\left(\otimes^{M} A, g\right)\left\|_{p} \leq\right\| S\right|(A, g)\right\|_{p}$. The converse inequality is a special case of (iv) we treat now.

(iv) We first perform a unitary permutation of the coordinates, which is justified by part (ii), so that we may assume that $B=\left(a_{l k}\right)_{1 \leq l, k \leq d^{\prime}}$. Next we consider the unitary map $U$ which maps the unit vector $e_{k}$ to $e_{k}$ if $k=1, \ldots, d^{\prime}$ and to $-e_{k}$ if $k=d^{\prime}+1, \ldots, d$. From (ii) it follows that, for $C:=(1 / 2)\left(A+U^{T} A U\right)$, one has $\left\|S\left|(C, g)\left\|_{p} \leq\right\| S\right|(A, g)\right\|_{p}$. The entries of $C$ satisfy $c_{l k}=a_{l k}$ if $l, k \in\left\{1, \ldots, d^{\prime}\right\}$ and $c_{l k}=0$ if $l \in\left\{1, \ldots, d^{\prime}\right\}$ and $k \notin\left\{1, \ldots, d^{\prime}\right\}$. Now the inequality $\left\|S\left|(B, g)\left\|_{p} \leq\right\| S\right|(C, g)\right\|_{p}$ can be proved by an appropriate augmentation of the Gaussian random variables and of the martingale difference sequence that has to be transformed: we add to each 
block $\phi_{(k-1) d^{\prime}+1}, \ldots, \phi_{k d^{\prime}}$ of Gaussian random variables $d-d^{\prime}$ independent Gaussian random variables to obtain a block size $d$ and add appropriate zero martingale differences to the original martingale difference sequence. Now the transformation with respect to $B$ can be artificially written as a transformation with respect to $C$.

The previous lemma will be used to prove the following result:

Proposition 3.7. (i) Assume that $A \in M(d, d)$ is real and symmetric, and denote by $\lambda_{\max }$ (respectively, $\lambda_{\min }$ ) the largest (respectively, the smallest) eigenvalue of $A$. Let $B \in M\left(d^{\prime}, d^{\prime}\right)$ be another real and symmetric matrix such that each eigenvalue $\lambda$ of $B$ satisfies $\lambda_{\min } \leq \lambda \leq \lambda_{\max }$. Then for any $S \in L(X, Y)$ and $p \in(1, \infty)$, it holds that

$$
\left\|S\left|(B, W)\left\|_{p} \leq\right\| S\right|(A, W)\right\|_{p} .
$$

(ii) Assume that $A \in M(d, d)$ is real and antisymmetric, and denote by $\lambda_{\max }$ (respectively, $\lambda_{\min }$ ) the largest (respectively, the smallest) eigenvalue of $i A$. Let $B \in M\left(d^{\prime}, d^{\prime}\right)$ be another real and antisymmetric matrix such that each eigenvalue $\lambda$ of $i B$ satisfies $\lambda_{\min } \leq \lambda \leq \lambda_{\max }$. Then for any $S \in L(X, Y)$ and $p \in(1, \infty)$, it holds that

$$
\left\|S\left|(B, W)\left\|_{p} \leq\right\| S\right|(A, W)\right\|_{p} .
$$

Proof. (i) By Lemma 3.6 (i) it is enough to show that $\|S \mid(B, g)\|_{p} \leq$ $\|S \mid(A, g)\|_{p}$. Moreover, by applying the spectral theorem for symmetric matrices and part (ii) of the same Lemma we may assume that both $A$ and $B$ are (real and) diagonal. In addition, by Lemma 3.6 (iii) we may replace $A$ by the tensor product $\otimes^{d^{\prime}} A$. Observe that the tensor product $\otimes^{d^{\prime}} A$ is diagonal and at least $d^{\prime}$ of its diagonal elements have the value $\lambda_{\max }$, and the same holds true for $\lambda_{\min }$. By applying again a unitary permutation for the coordinates and part (iv) of Lemma 3.6 we obtain for any given sequence $\Lambda:=\left(\lambda_{1}, \ldots, \lambda_{d^{\prime}}\right)$ with $\lambda_{j} \in\left\{\lambda_{\max }, \lambda_{\min }\right\}$ for all $1 \leq j \leq d^{\prime}$ that the diagonal matrix $A_{\Lambda} \in M\left(d^{\prime}, d^{\prime}\right)$ (with the diagonal $\Lambda$ ) satisfies

$$
\left\|S\left|\left(A_{\Lambda}, W\right)\left\|_{p} \leq\right\| S\right|(A, W)\right\|_{p} .
$$

By the assumption on the eigenvalues of the matrix $B$, we may express $B$ as a convex combination of matrices of the form $A_{\Lambda}$. This clearly yields the claim.

(ii) The matrix $i A$ is self-adjoint, so that the eigenvalues are real. A simple examination of the spectral decomposition of $i A$ (observe that $\lambda$ and $-\lambda$ are simultaneously eigenvalues for $i A$ ) we may write $A$, after a unitary transformation, in the form

$$
A=B_{1} \otimes \ldots \otimes B_{\ell},
$$

where each $B_{k}$ is of the form $B_{k}=c_{k} \mathrm{~A}_{\mathrm{as}}\left(\mathrm{A}_{\mathrm{as}}\right.$ was defined in (10)), with $\lambda_{\min } \leq c_{k} \leq \lambda_{\max }$ (in case $d$ is odd the last one, i.e. $B_{\ell}$, equals the $1 \times 1$ zero matrix and $\left.\lambda_{\min } \leq 0 \leq \lambda_{\max }\right)$. Now the claim follows by an extreme point argument like in (i). 
Proposition 3.8. Let $m$ be a homogeneous, even, and smooth multiplier on $\mathbf{R}^{d}, d \geq 2$. Then for any $S \in L(X, Y)$ it holds that

$$
\left\|T_{m} \otimes S: L_{X}^{p}\left(\mathbf{R}^{d}\right) \rightarrow L_{Y}^{p}\left(\mathbf{R}^{d}\right)\right\| \leq c\left\|T_{m_{0}} \otimes S: L_{X}^{p}\left(\mathbf{R}^{d}\right) \rightarrow L_{Y}^{p}\left(\mathbf{R}^{d}\right)\right\|
$$

for $S \in L(X, Y)$ and $p \in(1, \infty)$, where $c>0$ depends at most on $m$.

Proof. In the following we always assume that $\xi, \theta \in S^{d-1}$, so that (for example) $m_{0}(\xi)=2 \xi_{1}^{2}-1$. Let $a \in(0,1)$. By composing $m_{0}$ with the linear map $\xi \mapsto B_{a}(\xi):=\left(\sqrt{1-a^{2}} \xi_{1}, \xi_{2}, \ldots, \xi_{d}\right)$ we infer by $(7)$ that

$$
\left\|T_{m_{a}} \otimes S: L_{X}^{p}\left(\mathbf{R}^{d}\right) \rightarrow L_{Y}^{p}\left(\mathbf{R}^{d}\right)\right\| \leq \frac{1}{1+a}\left\|T_{m_{0}} \otimes S: L_{X}^{p}\left(\mathbf{R}^{d}\right) \rightarrow L_{Y}^{p}\left(\mathbf{R}^{d}\right)\right\|,
$$

where

$$
\begin{aligned}
m_{a}(\xi) & :=\frac{2-a^{2}}{2(1+a)}+\frac{a^{2}}{2(1+a)} m_{0} \circ B_{a}(\xi)=(1-a) \frac{1}{1-a^{2} \xi_{1}^{2}} \\
& =(1-a) \sum_{k=0}^{\infty} a^{2 k} \xi_{1}^{2 k}
\end{aligned}
$$

and we also use the estimate

$$
\left\|I d \otimes S: L_{X}^{p}\left(\mathbf{R}^{d}\right) \rightarrow L_{Y}^{p}\left(\mathbf{R}^{d}\right)\right\| \leq\|S\| \leq\left\|T_{m_{0}} \otimes S: L_{X}^{p}\left(\mathbf{R}^{d}\right) \rightarrow L_{Y}^{p}\left(\mathbf{R}^{d}\right)\right\|
$$

(for example, use Lemma 2.2 and $\left\|m_{0}\right\|_{\infty}=1$ ). Of course, given $\theta \in S^{d-1}$ we obtain the same estimates for

$$
m_{a}^{\theta}(\xi):=(1-a) \sum_{k=0}^{\infty} a^{2 k}\langle\xi, \theta\rangle^{2 k} .
$$

Given $r \in(0,1)$, the $d$-dimensional Poisson kernel for $B(0,1)$ has the form

$$
\begin{aligned}
P(r \xi, \theta) & =\frac{1}{\omega_{d-1}} \frac{\left(1-r^{2}\right)}{\left(1+r^{2}-2 r\langle\xi, \theta\rangle\right)^{d / 2}} \\
& =\frac{\left(1-r^{2}\right)\left(1+r^{2}\right)^{-d / 2}}{\omega_{d-1}}\left(1-\frac{2 r\langle\xi, \theta\rangle}{1+r^{2}}\right)^{-d / 2}
\end{aligned}
$$

with $\omega_{d-1}=|\partial B(0,1)|$. By the Taylor expansion of $y \mapsto(1-y)^{-d / 2}$ we obtain that

$$
\begin{aligned}
P^{s}(r \xi, \theta) & :=\frac{1}{2}(P(r \xi, \theta)+P(r \xi,-\theta)) \\
& =\frac{\left(1-r^{2}\right)\left(1+r^{2}\right)^{-d / 2}}{\omega_{d-1}} \sum_{k=0}^{\infty}\left(\frac{2 r}{1+r^{2}}\right)^{2 k}\langle\xi, \theta\rangle^{2 k}\left(\begin{array}{c}
-d / 2 \\
2 k
\end{array}\right) .
\end{aligned}
$$

Fix $\varepsilon>0$ and let

$$
c_{0}:=2 \omega_{d-1} \frac{\Gamma(\varepsilon+1) \Gamma(d / 2)}{\Gamma((d / 2)+\varepsilon)} .
$$

Using the substitution $u=2 r /\left(1+r^{2}\right)$ and Euler's $\beta$-integral yields that

$$
\begin{aligned}
& \int_{0}^{1}\left(1+r^{2}\right)^{-2+d / 2}\left(1-\frac{2 r}{1+r^{2}}\right)^{d / 2-1+\varepsilon} P^{s}(r \xi, \theta) d r \\
= & \frac{1}{2 \omega_{d-1}} \sum_{k=0}^{\infty}\left(\int_{0}^{1}(1-u)^{d / 2-1+\varepsilon} u^{2 k} d u\right)\left(\begin{array}{c}
-d / 2 \\
2 k
\end{array}\right)\langle\xi, \theta\rangle^{2 k}
\end{aligned}
$$




$$
\begin{aligned}
& =\frac{1}{2 \omega_{d-1}} \frac{\Gamma(d / 2+\varepsilon)}{\Gamma(d / 2)} \sum_{k=0}^{\infty}\left(\frac{\Gamma(d / 2+2 k)}{\Gamma(d / 2+2 k+1+\varepsilon)}\right)\langle\xi, \theta\rangle^{2 k} \\
& =\frac{1}{c_{0}} \int_{0}^{1}(1-a)^{\varepsilon-1} a^{d / 2-1} m_{a}^{\theta}(\xi) d a .
\end{aligned}
$$

Let us denote by $\operatorname{sh}(d)$ a complete orthonormal $L^{2}$-basis of $L^{2}\left(S^{d-1}, \lambda\right)$, where $\lambda$ is the normalized Haar measure, consisting of spherical harmonics on $S^{d}$ (we refer to $[23,24]$ for more details on spherical harmonics). For $\psi \in \operatorname{sh}(d)$ we denote by $\operatorname{deg}(\psi)$ the degree of $\psi$. Because the function $(r, \xi) \mapsto r^{k} \psi(\xi)$ is harmonic if $\operatorname{deg}(\psi)=k$ we have in this case

$$
\int_{S^{d-1}} P(r \xi, \theta) \psi(\theta) d \theta=r^{k} \psi(\xi) .
$$

Define

$$
\lambda_{k}:=c_{0} \int_{0}^{1}\left(1+r^{2}\right)^{-2+d / 2}\left(1-\frac{2 r}{1+r^{2}}\right)^{d / 2-1+\varepsilon} r^{k} d r \sim_{c(\varepsilon, d)} k^{1-d-2 \varepsilon} .
$$

Let $a_{\psi}:=\langle m, \psi\rangle / \lambda_{k}$ if $\psi \in \operatorname{sh}(d)$ and $\operatorname{deg}(\psi)=k$. Applying [23, p. 70] gives

$$
\sum_{\psi \in \mathbf{s h}_{d}}\left|a_{\psi}\right|^{2}<\infty
$$

Because $m$ is even we find an even $f \in L^{2}\left(S^{d-1}, \lambda\right)$ with $\langle f, \psi\rangle=a_{\psi}$, so that

$$
m=\sum_{k=0}^{\infty} \lambda_{k} \sum_{\psi \in \mathbf{s h}_{d}, \operatorname{deg}(\psi)=k}\langle f, \psi\rangle \psi
$$

in $L^{2}\left(S^{d-1}, \lambda\right)$. If we can show that

$$
m(\xi)=\int_{S^{d-1}} \int_{0}^{1}(1-a)^{\varepsilon-1} a^{\frac{d}{2}-1} m_{a}^{\theta}(\xi) f(\theta) d a d \theta
$$

for $\xi \in S^{d-1}$, then this would give our assertion since

$$
\int_{S^{d-1}} \int_{0}^{1}(1-a)^{\varepsilon-1} a^{\frac{d}{2}-1}|f(\theta)| d a d \theta<\infty
$$

implies that $m$ is a convex combination of the multipliers $m_{a}^{\theta}$. In order to verify equality (16) it is sufficient to show that

$$
\begin{aligned}
\int_{S^{d-1}}\left[\int_{S^{d-1}} \int_{0}^{1}\right. & \left.(1-a)^{\varepsilon-1} a^{\frac{d}{2}-1} m_{a}^{\theta}(\xi) f(\theta) d a d \theta\right] \bar{\psi}(\xi) d \xi \\
& =c_{0} \int_{0}^{1}\left(1+r^{2}\right)^{-2+d / 2}\left(1-\frac{2 r}{1+r^{2}}\right)^{d / 2-1+\varepsilon} r^{k} d r\langle f, \psi\rangle
\end{aligned}
$$

for $\psi \in \mathbf{s h}_{d}$ with $\operatorname{deg}(\psi)=k$. But this follows by a computation from (13) and (14).

Remark 3.9. A slight additional argument shows that one may allow any even multiplier $m$ in the above proposition such that $m_{\mid S^{d-1}} \in W^{s, 1}\left(S^{d-1}\right)$ for some $s>d-1$. Especially, if $d=2$ this class contains all $m$ such that for some $\varepsilon>0$ the derivative $D^{\varepsilon} m_{\mid S^{1}}$ is a function of bounded variation. 
We are ready for

Proof of Theorem 3.1. We first verify that $\operatorname{UMD}_{p}(S)$ is comparable to the corresponding norm of the operator $T_{m_{0}} \otimes S$.

Observe that $\max _{|\xi|=1} m_{0}(\xi)=1$ and $\min _{|\xi|=1} m_{0}(\xi)=-1$. An application of Proposition 3.4 yields that

$$
\operatorname{UMD}_{p}(S) \leq\left\|T_{m_{0}} \otimes S: L_{X}^{p}\left(\mathbf{R}^{d}\right) \rightarrow L_{Y}^{p}\left(\mathbf{R}^{d}\right)\right\| .
$$

By Proposition 3.5 we have in turn

$$
\left\|T_{m_{0}} \otimes S: L_{X}^{p}\left(\mathbf{R}^{d}\right) \rightarrow L_{Y}^{p}\left(\mathbf{R}^{d}\right)\right\| \leq\left\|S \mid\left(A_{\mathrm{s}, d}, W\right)\right\|_{p} .
$$

The eigenvalues of $A_{\mathrm{s}, d}$ and $\mathrm{A}_{\mathrm{s}}$ are \pm 1 . Hence Proposition 3.7 (i) and Lemma 3.6 (i) yield that

$$
\left\|S\left|\left(A_{\mathrm{s}, d}, W\right)\left\|_{p} \leq\right\| S\right|\left(\mathrm{A}_{\mathrm{s}}, W\right)\right\|_{p}=\left\|S \mid\left(\mathrm{A}_{\mathrm{s}}, g\right)\right\|_{p} .
$$

However, the $A_{\mathrm{s}}$-transform of discrete Gaussian martingales is a special case of a UMD-martingale transform and hence in case $S=$ Id it follows immediately that

$$
\left\|S \mid\left(\mathrm{A}_{\mathrm{s}}, g\right)\right\|_{p} \leq \operatorname{UMD}_{p}(S) .
$$

There are various ways to check this inequality for general $S$ (note that we started with Walsh-Paley martingales in the definition of $\operatorname{UMD}_{p}(S)$ ). An easy self-consistent way would be to apply the central limit theorem argument from Lemma 4.2 to the Bernoulli variables to replace the Bernoulli variables by the Gaussian random variables (there are arguments to switch from Walsh-Paley martingale difference sequences to arbitrary martingale difference sequences; see for example [10, p. 12] and [20]). By combining the inequalities (17)-(20) we obtain that

$$
\operatorname{UMD}_{p}(S)=\left\|T_{m_{0}} \otimes S: L_{X}^{p}\left(\mathbf{R}^{d}\right) \rightarrow L_{Y}^{p}\left(\mathbf{R}^{d}\right)\right\|=\left\|S \mid\left(\mathrm{A}_{\mathrm{s}}, W\right)\right\|_{p} .
$$

This proves the equalities in the theorem.

Now let us assume that $A \in M(d, d)$ is symmetric and non-trivial, and that $m$ is an even, smooth, and non-trivial multiplier. Proposition 3.4 shows that $\operatorname{UMD}_{p}(S)$ is dominated by a multiple of the norm of $T_{m} \otimes S$, and Proposition 3.8 verifies that this norm in turn is dominated by a multiple of the norm of $T_{m_{0}} \otimes S$. The linear equivalence of the norm of $T_{m} \otimes S$ to the UMD-constant of $X$ follows now from (21).

Finally, the equivalence of $\operatorname{UMD}_{p}(S)$ to $\|S \mid(A, W)\|_{p}$ will be deduced from (21) by an application of Proposition 3.7 (i). Denote by $\lambda_{\min }, \lambda_{\max }$ the minimal and maximal eigenvalue of $A$. Firstly, we obtain that

$$
\left\|S\left|(A, W)\left\|_{p} \leq c\right\| S\right|\left(\mathrm{A}_{\mathrm{s}}, W\right)\right\|_{p}
$$

with $c:=\max \left(\left|\lambda_{\max }\right|,\left|\lambda_{\min }\right|\right)$. To consider the other direction, let $\alpha:=$ $2 /\left(\lambda_{\max }-\lambda_{\min }\right)$ and $\beta:=-\left(\lambda_{\max }+\lambda_{\min }\right) /\left(\lambda_{\max }-\lambda_{\min }\right)$, so that $B:=\alpha A+\beta$ satisfies $\lambda_{\max }(B)=-\lambda_{\min }(B)=1$. Consequently,

$$
\begin{aligned}
\left\|S \mid\left(\mathrm{A}_{\mathrm{s}}, W\right)\right\|_{p} & \leq \alpha\left\|S\left|(A, W)\left\|_{p}+\beta\right\| S\right|(I d, W)\right\|_{p} \\
& \leq \alpha\left\|S\left|(A, W)\left\|_{p}+\frac{\beta}{\max \left\{\left|\lambda_{\max }\right|,\left|\lambda_{\min }\right|\right\}}\right\| S\right|(A, W)\right\|_{p}
\end{aligned}
$$

and the proof of Theorem 3.1 is complete. 
Remark 3.10. There is a shorter argument for part of Theorem 3.1 which, modulo some 'hand waving', bypasses Lemma 3.6 and Proposition 3.7. Namely, in order to prove e.g. the statement

$$
\operatorname{UMD}_{p}(X)=\left\|T_{m_{0}}: L_{X}^{p}\left(\mathbf{R}^{d}\right) \rightarrow L_{Y}^{p}\left(\mathbf{R}^{d}\right)\right\| \sim_{C}\left\|T_{m}: L_{X}^{p}\left(\mathbf{R}^{d}\right) \rightarrow L_{Y}^{p}\left(\mathbf{R}^{d}\right)\right\|
$$

one only needs Propositions 3.4, 3.5, and 3.8 and $\left\|S \mid\left(A_{\mathrm{s}, d}, W\right)\right\|_{p} \leq$ $\mathrm{UMD}_{p}(S)$. Because $A_{\mathrm{s}, d}$ is a diagonal matrix, the latter inequality can be suitably approximated by a UMD-transform inequality.

\section{OdD MUltipliers}

A canonical example of an odd multiplier is the Hilbert transform. In this section we verify that all odd multipliers, as well as all antisymmetric transforms for stochastic integrals are linearly comparable to the Hilbert transform. Observe that an odd multiplier maps real functions to purely imaginary ones, whence we consider below the operator $T_{i m}$ in order to be able to allow also real Banach spaces in the statement.

Theorem 4.1. Assume that $m \in C^{\infty}\left(\mathbf{R}^{d} \backslash\{0\}\right), d \geq 2$, is a real non-zero, homogeneous, and odd multiplier, and that $A \in M(d, d)$ is a non-zero and anti-symmetric matrix. Let $p \in(1, \infty)$. Then there is a constant $C=$ $C(A, m)$ such that for every pair of Banach spaces $X$ and $Y$ and for every operator $S \in L(X, Y)$ one has

$$
\begin{aligned}
\left\|T_{i m} \otimes S: L_{X}^{p}\left(\mathbf{R}^{d}\right) \rightarrow L_{Y}^{p}\left(\mathbf{R}^{d}\right)\right\| & \sim_{C}\left\|S\left|(A, W)\left\|_{p} \sim_{C}\right\| S\right|\left(\mathrm{A}_{\mathrm{as}}, W\right)\right\|_{p} \\
& =\left\|\mathcal{H} \otimes S: L_{X}^{p}(\mathbf{R}) \rightarrow L_{Y}^{p}(\mathbf{R})\right\| .
\end{aligned}
$$

We start the proof by

Lemma 4.2. For $p \in(1, \infty)$ and $S \in L(X, Y)$ one has that

$$
\left\|S \mid\left(\mathrm{A}_{\mathrm{as}}, W\right)\right\|_{p} \leq\left\|\widetilde{\mathcal{H}} \otimes S: L_{X}^{p}(\mathbf{T}) \rightarrow L_{Y}^{p}(\mathbf{T})\right\| .
$$

Proof. Assume that $M \geq 1$ and that $a_{k}, b_{k}:[-1,1]^{2 k-2} \rightarrow X, k=$ $1, \ldots, M$, are bounded and continuous. Lemma 3.3 yields that

$$
\begin{aligned}
& \quad \| \sum_{k=1}^{M}\left(\widetilde{\mathcal{H}} \sin \left(\theta_{k}\right) S a_{k}\left(\sin \left(\theta_{1}\right), \ldots, \sin \left(\theta_{k-1}\right) ; \cos \left(\theta_{1}\right), \ldots, \cos \left(\theta_{k-1}\right)\right)\right. \\
& \quad+\widetilde{\mathcal{H}} \cos \left(\theta_{k}\right) S b_{k}\left(\sin \left(\theta_{1}\right), \ldots, \sin \left(\theta_{k-1}\right) ; \cos \left(\theta_{1}\right), \ldots, \cos \left(\theta_{k-1}\right)\right) \|_{L_{p}^{Y}} \\
& \leq\left\|\widetilde{\mathcal{H}} \otimes S: L_{p}^{X}(\mathbf{T}) \rightarrow L_{p}^{Y}(\mathbf{T})\right\| \times \\
& \quad \times \| \sum_{k=1}^{M}\left(\sin \left(\theta_{k}\right) a_{k}\left(\sin \left(\theta_{1}\right), \ldots, \sin \left(\theta_{k-1}\right) ; \cos \left(\theta_{1}\right), \ldots, \cos \left(\theta_{k-1}\right)\right)\right. \\
& \quad+\cos \left(\theta_{k}\right) b_{k}\left(\sin \left(\theta_{1}\right), \ldots, \sin \left(\theta_{k-1}\right) ; \cos \left(\theta_{1}\right), \ldots, \cos \left(\theta_{k-1}\right)\right) \|_{L_{p}^{X}} .
\end{aligned}
$$

Next, we apply a blocking argument. Let $N, L \geq 1$ be integers and assume that $A_{k}, B_{k}:[-1,1]^{2 k-2} \rightarrow X, k=1, \ldots, N$, are bounded and continuous functions. For $M=N L$ we apply (22) to

$$
a_{(k-1) L+j}\left(\sin \left(\theta_{1}\right), \ldots, \sin \left(\theta_{(k-1) L+j-1}\right) ; \cos \left(\theta_{1}\right), \ldots, \cos \left(\theta_{(k-1) L+j-1}\right)\right)
$$




$$
\begin{gathered}
=A_{k}\left((L / 2)^{-1 / 2}\left(\sin \left(\theta_{1}\right)+\ldots+\sin \left(\theta_{L}\right)\right)\right. \\
(L / 2)^{-1 / 2}\left(\sin \left(\theta_{L+1}\right)+\ldots+\sin \left(\theta_{2 L}\right)\right) \\
\ldots,(L / 2)^{-1 / 2}\left(\sin \left(\theta_{(k-2) L+1}\right)+\ldots+\sin \left(\theta_{(k-1) L}\right)\right) \\
(23) \quad(L / 2)^{-1 / 2}\left(\cos \left(\theta_{1}\right)+\ldots+\cos \left(\theta_{L}\right)\right) \\
(L / 2)^{-1 / 2}\left(\cos \left(\theta_{L+1}\right)+\ldots+\cos \left(\theta_{2 L}\right)\right) \\
\left.\ldots,(L / 2)^{-1 / 2}\left(\cos \left(\theta_{(k-2) L+1}\right)+\ldots+\cos \left(\theta_{(k-1) L}\right)\right)\right)
\end{gathered}
$$

and with an analogous choice for the coefficients $b_{(k-1) L+j}$ for $1 \leq k \leq N$ and $1 \leq j \leq L$. By taking into account the action of $\widetilde{\mathcal{H}}$ on the trigonometric polynomials we obtain that

$$
\begin{gathered}
\| \sum_{k=1}^{N}\left(-S c_{k} A_{k}\left(s_{1}, \ldots, s_{k-1} ; c_{1}, \ldots, c_{k-1}\right)+\right. \\
\left.+S s_{k} B_{k}\left(s_{1}, \ldots, s_{k-1} ; c_{1}, \ldots, c_{k-1}\right)\right) \|_{L_{p}^{Y}} \\
\leq\left\|\widetilde{\mathcal{H}} \otimes S: L_{p}^{X}(\mathbf{T}) \rightarrow L_{p}^{Y}(\mathbf{T})\right\| \times \\
\quad \times \| \sum_{k=1}^{N}\left(s_{k} A_{k}\left(s_{1}, \ldots, s_{k-1} ; c_{1}, \ldots, c_{k-1}\right)+\right. \\
\left.+c_{k} B_{k}\left(s_{1}, \ldots, s_{k-1} ; c_{1}, \ldots, c_{k-1}\right)\right) \|_{L_{p}^{X}}
\end{gathered}
$$

where we have used the abbreviations $s_{k}:=(L / 2)^{-1 / 2} \sum_{j=1}^{L} \sin \left(t_{(k-1) L+j}\right)$ and $c_{k}:=(L / 2)^{-1 / 2} \sum_{j=1}^{L} \cos \left(t_{(k-1) L+j}\right)$ for $k=1, \ldots, N$. By keeping $N$ fixed, letting $L \rightarrow \infty$, and by applying the central limit theorem (here we may normalize the Lebesgue measure on $\mathbf{T}$ ) and the fact that cos and $\sin$ are uncorrelated, one gets that

$$
\left\|S \mid\left(\mathrm{A}_{\mathrm{as}}, g\right)\right\|_{p} \leq\left\|\tilde{\mathcal{H}} \otimes S: L_{X}^{p}(\mathbf{T}) \rightarrow L_{Y}^{p}(\mathbf{T})\right\| .
$$

Finally, Lemma 3.6 verifies that $\left\|S\left|\left(\mathrm{~A}_{\text {as }}, g\right)\left\|_{p}=\right\| S\right|\left(\mathrm{A}_{\text {as }}, W\right)\right\|_{p}$ and we are done.

The following lemma is well-known. For the convenience of the reader we recall the idea of its proof.

Lemma 4.3. For $p \in(1, \infty)$ one has that

$$
\left\|\mathcal{H} \otimes S: L_{X}^{p}(\mathbf{R}) \rightarrow L_{Y}^{p}(\mathbf{R})\right\| \leq\left\|S \mid\left(\mathrm{A}_{\mathrm{as}}, W\right)\right\|_{p} .
$$

Proof. $\quad$ We use $\left\|\mathcal{H} \otimes S: L_{X}^{p}(\mathbf{R}) \rightarrow L_{Y}^{p}(\mathbf{R})\right\|=\| \widetilde{\mathcal{H}} \otimes S: L_{X, 0}^{p}(\mathbf{T}) \rightarrow$ $L_{Y}^{p}(\mathbf{T}) \|$ from Lemma 2.2 and consider $f(\theta):=\sum_{k=1}^{n}\left(\sin (k \theta) x_{k}+\cos (k \theta) y_{k}\right)$ with $x_{k}, y_{k} \in X$ as a function on the unit circle. Then, a.s.,

$$
u\left(W_{\tau}\right)=\int_{0}^{\tau} \nabla u\left(W_{t}\right) \cdot d W_{t} \text { and } v\left(W_{\tau}\right)=-\int_{0}^{\tau} \nabla u\left(W_{t}\right) \cdot d\left(\mathrm{~A}_{\mathrm{as}} W\right)_{t}
$$


by Itô's formula, where $u$ and $v$ are the harmonic extensions of $f$ and $\sum_{k=1}^{n}\left(-\cos (k \theta) x_{k}+\sin (k \theta) y_{k}\right)$ to the unit disc, $\left(W_{t}\right)_{t \geq 0}$ is a standard twodimensional standard Brownian motion, and $\tau:=\inf \left\{t \geq 0:\left|W_{t}\right|=1\right\}$.

Proof of Theorem 4.1. The equality

$$
\left\|S \mid\left(\mathrm{A}_{\mathrm{as}}, W\right)\right\|_{p}=\left\|\mathcal{H} \otimes S: L_{X}^{p}(\mathbf{R}) \rightarrow L_{Y}^{p}(\mathbf{R})\right\|
$$

follows from Lemmas 4.2, 4.3, and 2.2. Moreover,

$$
\left\|S\left|(A, W)\left\|_{p} \sim_{C}\right\| S\right|\left(\mathrm{A}_{\mathrm{as}}, W\right)\right\|_{p}
$$

is a consequence of Proposition 3.7 (ii) (note, that the eigenvalues of $i \mathrm{~A}_{\mathrm{as}}$ are \pm 1 and that $i A$ has at least two symmetric non-zero real eigenvalues). Moreover, by a classical argument called 'the method of rotations' (see e.g. [15, p.271, formula (4.2.20)] one may express any odd (and smooth) multiplier operator $T_{i m}$ as an average of directional Hilbert-transforms which immediately yields that the norm of $T_{i m} \otimes S$ is linearly dominated by that of $\mathcal{H} \otimes S$. Finally, if $m$ is an odd and non-zero multiplier, we may assume that $m\left(e_{1}\right)=1$. The corresponding discrete multiplier $\widetilde{m}$ satisfies $-i \widetilde{m}\left(k_{1} e_{1}\right)=-i \operatorname{sgn}\left(k_{1}\right)$ for $k_{1} \neq 0$ and $-i \widetilde{m}\left(0 e_{1}\right)=0$. By the consideration of $\widetilde{f}\left(\theta_{1}, \ldots, \theta_{d}\right)=f\left(\theta_{1}\right):=\sum_{k=1}^{n}\left(\sin \left(k_{1} \theta_{1}\right) x_{k_{1}}+\cos \left(k_{1} \theta_{1}\right) y_{k_{1}}\right)$ with $x_{k_{1}}, y_{k_{1}} \in X$ and observing that $((\widetilde{H} \otimes S) f)\left(\theta_{1}\right)=\left(\left(-T_{i \widetilde{m}} \otimes S\right) \widetilde{f}\right)\left(\theta_{1}, \ldots, \theta_{d}\right)$ we immediately get that

$$
\left\|\widetilde{H} \otimes S: L_{X, 0}^{p}(\mathbf{T}) \rightarrow L_{Y}^{p}(\mathbf{T})\right\| \leq\left\|T_{i \widetilde{m}} \otimes S: L_{X}^{p}\left(\mathbf{T}^{d}\right) \rightarrow L_{Y}^{p}\left(\mathbf{T}^{d}\right)\right\|
$$

so that $\left\|H \otimes S: L_{X}^{p}(\mathbf{R}) \rightarrow L_{Y}^{p}(\mathbf{R})\right\| \leq\left\|T_{i m} \otimes S: L_{X}^{p}\left(\mathbf{R}^{d}\right) \rightarrow L_{Y}^{p}\left(\mathbf{R}^{d}\right)\right\|$ by Lemma 2.2 .

\section{AdDitional REMARKS}

The remaining main open problem is whether or not we have the linear equivalence of the norms

$$
\operatorname{UMD}_{p}(S) \sim\left\|\mathcal{H} \otimes S: L_{X}^{p}(\mathbf{R}) \rightarrow L_{Y}^{p}(\mathbf{R})\right\| .
$$

By the results obtained in Theorems 3.1 and 4.1 this problem can be formulated now in various ways, for example in a purely probabilistic way via martingale transforms or via multipliers. To find counterexamples to (24) in the category of Banach spaces (i.e. $S=I d_{X}$ for a Banach space $X$ ) seems to be harder than to find counterexamples for operators $S \in L(X, Y)$, which is one reason for our usage of the operator setting. At the same time the operator setting gives an easier control whether or not estimates are linear (for example $\operatorname{UMD}_{p}\left(I d_{X}\right)<\infty$ if and only if $\left\|\mathcal{H} \otimes I d_{X}: L_{X}^{p}(\mathbf{R}) \rightarrow L_{X}^{p}(\mathbf{R})\right\|<\infty$ because of $I d_{X}^{2}=I d_{X}$ which does not work for operators). Natural candidates to disprove (24) in the setting of operators are the operators of summation $\sigma_{n}: \ell_{n}^{1} \rightarrow \ell_{n}^{\infty}$. For them it is known that

$$
\left\|\mathcal{H} \otimes \sigma_{n}: L_{\ell_{n}^{1}}^{2}(\mathbf{R}) \rightarrow L_{\ell_{n}^{\infty}}^{2}(\mathbf{R})\right\| \sim \log (n+1),
$$


see [13] (Section IV, Satz 2.1 (proof) and Korollar 2.4) (cf. also [22] (2.4.4)). So far, the best estimates for the UMD-constants are

$$
\frac{1}{c} \sqrt{\log (n+1)} \leq \mathrm{UMD}_{2}\left(\sigma_{n}\right) \leq c \log (n+1)
$$

where the lower estimate follows from [14] and the upper one is a consequence of $\mathrm{UMD}_{2}\left(I d_{\ell_{n}^{\infty}}\right) \leq c \log (n+1)$ which is folklore.

A determination of the UMD-constant of $\sigma_{n}$ would be of interest for several reasons: in case of $\mathrm{UMD}_{2}\left(\sigma_{n}\right) \sim \log (n+1)$ this would imply that each non-superreflexive Banach space $X$ contains $n$-dimensional subspaces $E_{n}$ such that the lower bound $\mathrm{UMD}_{2}\left(I d_{E_{n}}\right) \geq \log (n+1) / c$ holds, because due to R.C. James a Banach space $X$ is non-superreflexive if and only if the operators $\sigma_{n}$ can be uniformly factorized through certain $n$-dimensional subspaces $E_{n} \subseteq X$ (see [18]). Having the lower estimate one might ask for more connections between the quantitative behavior of the UMD-constants of the finite dimensional subspaces of a Banach space and the property that the space is non-superreflexive. On the other hand, any estimate of type $\mathrm{UMD}_{2}\left(\sigma_{n}\right)=o(\log (n+1))$ would offer some new inside into martingale transforms.

\section{Appendix}

We recall the well-known connection between singular integrals and $A$ transforms of stochastic integrals corresponding to heat extensions of functions to the upper half space. A simple proof will be sketched below for the readers convenience. We refer to [2] for the original result, and to [4] and the references therein for corresponding results that use the harmonic extension instead. In order to state the relation let $d \geq 1$ and assume a real matrix $A=\left[a_{k l}\right] \in M(d, d)$. We define the operator $U_{A}$ for smooth elements $f, g \in C_{0}^{\infty}\left(\mathbf{R}^{d}\right)$ through the bilinear form

$$
\begin{aligned}
& \int_{\mathbf{R}^{d}}\left(U_{A} f\right)(x) g(x) d x \\
:= & \lim _{T \rightarrow \infty}(2 \pi T)^{d / 2} \mathbf{E}\left[\left(\int_{0}^{T} \nabla u\left(W_{t}, T-t\right) \cdot d(A W)_{t}\right) \times\right. \\
& \left.\times\left(\int_{0}^{T} \nabla v\left(W_{t}, T-t\right) \cdot d W_{t}\right)\right],
\end{aligned}
$$

where $u$ and $v$ are the heat extensions of $f$ and $g$, respectively, to the upper half space $\mathbf{R}^{d} \times \mathbf{R}_{+}$(i.e. $u(t, x):=\mathbb{E} f\left(x+W_{t}\right)$ and similarly for $\left.v\right), \nabla$ denotes the differentiation with respect to the $x$-variables, and $\left(W_{t}\right)_{t \geq 0}$ is a standard $d$-dimensional Brownian motion starting at the origin. Hence, for example, $u_{t}=\frac{1}{2} \Delta u$ and $u(x, 0)=f(x)$ for $x \in \mathbf{R}^{d}$.

Lemma 6.1. Let $p \in(1, \infty)$ and $d \geq 2$. The operator $U_{A}$ is well-defined and extends to a bounded operator on $L^{p}\left(\mathbf{R}^{d}\right)$ which can be expressed in terms of the Riesz transforms as

$$
U_{A}=-\sum_{k, l=1}^{d} a_{k l} R_{k} R_{l} .
$$


Proof. We let $f, g \in C_{0}^{\infty}\left(\mathbf{R}^{d}\right)$. From Itô's isometry we obtain that

$$
\begin{aligned}
& (2 \pi T)^{d / 2} \mathbf{E}\left[\left(\int_{0}^{T} \nabla u\left(W_{t}, T-t\right) \cdot d(A W)_{t}\right) \times\right. \\
& \left.\times\left(\int_{0}^{T} \nabla v\left(W_{t}, T-t\right) \cdot d W_{t}\right)\right] \\
= & (2 \pi T)^{d / 2} \mathbf{E} \int_{[0, T]}\left\langle A^{T} \nabla u\left(W_{t}, T-t\right), \nabla v\left(W_{t}, T-t\right)\right\rangle d t \\
= & \int_{\mathbf{R}^{d} \times[0, T]}\left\langle A^{T} \nabla u(x, t), \nabla v(x, t)\right\rangle(2 \pi T)^{d / 2} d \mu_{t, T}(x) d t,
\end{aligned}
$$

where $\mu_{t, T}=\operatorname{law}\left(W_{T-t}\right)$. Because of $\sup _{x \in \mathbf{R}^{d}, t>0} t^{\frac{d+1}{2}}|\nabla u(x, t)|<\infty$ and similarly for $v$ and by splitting the integration over $\mathbf{R}^{d} \times[0, T]$ into $\mathbf{R}^{d} \times$ $[0, T / 2]$ and $\mathbf{R}^{d} \times(T / 2, T]$ we see by standard arguments that

$$
\begin{aligned}
& \lim _{T \rightarrow \infty}(2 \pi T)^{d / 2} \mathbf{E}\left[\left(\int_{0}^{T} \nabla u\left(W_{t}, T-t\right) \cdot d(A W)_{t}\right) \times\right. \\
& \left.\times\left(\int_{0}^{T} \nabla v\left(W_{t}, T-t\right) \cdot d W_{t}\right)\right]=\int_{\mathbf{R}^{d} \times[0, \infty)}\left\langle A^{T} \nabla u(x, t), \nabla v(x, t)\right\rangle d x d t
\end{aligned}
$$

(note that $\int_{\mathbf{R}^{d} \times[0, \infty)}|\nabla u(x, t)|^{2} d x d t<\infty$ and the same for $v$, which follows by the argument below, cf. also [21, Lemma 1.1]). If $\mathcal{F} u$ is the Fourier transform of $u$ with respect to the $x$-variables, then it is well-known that $\mathcal{F} u(\xi, t)=(\mathcal{F} f)(\xi) e^{-t|\xi|^{2} / 2}$ for $\xi \in \mathbf{R}^{d}$ and $t>0$. Observe also that $\mathcal{F}\left(\left(d / d x_{k}\right) u\right)=i \xi_{k} \mathcal{F} u$ for $k=1, \ldots, d$. By Parseval's formula and Fubini's theorem we may compute

$$
\begin{aligned}
& \int_{\mathbf{R}^{d}}\left(U_{A} f\right)(x) g(x) d x \\
= & (2 \pi)^{-d} \int_{\mathbf{R}^{d}}\left(\int_{\mathbf{R}^{+}} e^{-t|\xi|^{2}} d t\right) \mathcal{F} f(\xi) \overline{\mathcal{F} g(\xi)}\langle i \xi, A(i \xi)\rangle d \xi \\
= & (2 \pi)^{-d} \int_{\mathbf{R}^{d}} \mathcal{F} f(\xi) \overline{\mathcal{F} g(\xi)}|\xi|^{-2}\langle\xi, A \xi\rangle d \xi \\
= & \int_{\mathbf{R}^{d}}\left(T_{m} f\right)(x) g(x) d x,
\end{aligned}
$$

where $m$ is the multiplier $m(\xi):=|\xi|^{-2}\langle\xi, A \xi\rangle$. By recalling that $R_{j}$ corresponds to the multiplier $\xi_{j} /(i|\xi|)$ the claim follows immediately.

\section{REFERENCES}

[1] R. Bañuelos and G. Wang: Sharp inequalities for martingales with applications to the Beurling-Ahlfors and Riesz transforms, Duke Math. J. 80 (1995), 575-600.

[2] R. Bañuelos and P. J. Méndez-Hernández: Space-time Brownian motion and the Beurling-Ahlfors transform, Indiana Univ. Math. J. 52 (2003), no. 4, 981-990.

[3] Al. Baernstein and S. Montgomery-Smith: Some conjectures about integral means of $\partial f$ and $\partial^{-} f$, Complex Analysis and Differential Equations, edited by C.Kiselman, Acta Universitatis Upsaliensis C., Volume 64 (1999), 92-109.

[4] R.F. Bass: Probabilistic Techniques in Analysis, Springer, 1995. 
[5] J. Bourgain: Some remarks on Banach spaces in which martingale difference sequences are unconditional, Ark. Mat., 21:163-168, 1983.

[6] J. Bourgain: Vector-valued singular integrals and the $H^{1}-B M O$ duality. In: Probability theory and harmonic analysis (Cleveland, Ohio, 1983), 1-19, Monogr. Textbooks Pure Appl. Math., 98, Dekker, New York, 1986.

[7] D. L. Burkholder : A geometrical characterization of Banach spaces in which martingale difference sequences are unconditional, Ann. Probab. 9 (1981), 997-1011.

[8] D. L. Burkholder : A geometric condition that implies the existence of certain singular integrals of Banach-space-valued functions, in: Conference on harmonic analysis in honor of Antoni Zygmund, Vol. I, II (Chicago, Ill., 1981), 270-286, Wadsworth Math. Ser., Wadsworth, Belmont, CA, 1983.

[9] D.L. Burkholder : Boundary value problems and sharp inequalities for martingale transforms, Ann. Prob. 12 (1984), 647-702.

[10] D.L. Burkholder : Explorations in martingale theory and its applications, Ecole d'Eté de Probabilités de Saint-Flour, XIX-1989, Lect. Notes Math. 1464, 1-66, 1992, Springer.

[11] D. L. Burkholder : Martingales and singular integrals in Banach spaces, in: Handbook of the geometry of Banach spaces, Vol. I, 233-269, North-Holland, Amsterdam, 2001.

[12] R. R. Coifman and G. Weiss: Transference Methods in Analysis, C.B.M.S. Regional Conference Series in Math. No. 31, Amer. Math. Soc., Providence, RI, 1976.

[13] M. Defant: Zur vektorwertigen Hilberttransformation, PhD thesis, Universität Kiel, 1986.

[14] S. Geiss: A counterexample concerning the relation between decoupling constants and UMD-constants, Trans. Amer. Soc., 351(4):1355-1375, 1999.

[15] L. Grafakos: Classical and modern Fourier analysis, Pearson 2004.

[16] R. F. Gundy and N. Varopoulos: Les transformations de Riesz et les int??grales stochastiques, C. R. Acad. Sci. Paris Sér. A-B 289 (1979), A13-A16.

[17] T.P. Hytönen: Aspects of probabilistic Littlewood-Paley theory in Banach spaces, Banach spaces and their applications in analysis, de Gruyter, Berlin, 343-355, 2007.

[18] R.C. James: Super-reflexive Banach spaces, Can. J. Math., 5 (1972), 896-904.

[19] K. de Leeuw: On $L_{p}$ multipliers, Ann. of Math 81 (1965), 364-379.

[20] B. Maurey: Système de Haar, Seminaire Maurey-Schwartz, Ecole Polytechnique, Paris, 1974-1975.

[21] S. Petermichl and A. Volberg: Heating of the Ahlfors-Beurling operator: weakly quasiregular maps on the plane are quasiregular, Duke Math. J., 112(2):281-305, 2003.

[22] A. Pietsch and J. Wenzel: Orthonormal systems and Banach space geometry, Cambridge University Press, 1998.

[23] E. M. Stein: Singular integrals and differentiability properties of functions, Princeton University Press 1970.

[24] E. M. Stein and G. Weiss: Introduction to Fourier analysis on Euclidean spaces, Princeton University Press 1971.

[25] A. Volberg and F. Nazarov: Heat extension of the Beurling operator and estimates for its norm, (Russian. Russian summary), Algebra i Analiz 15 (2003), 142-158; translation in St. Petersburg Math. J. 15 (2004), 563-573.

[26] L. Weis: Operator-valued Fourier multiplier theorems and maximal $L_{p}$-regularity, Math. Ann. 319 (2001), 735-758.

University of Jyväskylä, Department of Mathematics and Statistics, P.O.

Box 35 (MaD), FIN-40014 University of Jyväskylä, Finland

E-mail address: geiss@maths.jyu.fi

University of Missouri, Mathematics Department, Columbia, MO 65211 USA

E-mail address: stephen@math.missouri.edu

University of Helsinki, Department of Mathematics and Statistics, P.O.

Box 68, Fin-00014 University of Helsinki, Finland

E-mail address: eero.saksman@helsinki.fi 